\title{
ASYMPTOTIC BEHAVIOUR OF MOMENTS AND LOCAL DIMENSIONS OF MEASURES
}

\author{
Lars Olsen \\ University of St. Andrews, Department of Mathematics \\ St. Andrews, Fife KY16 9SS, Scotland; lo@st-and.ac.uk
}

\begin{abstract}
We analyse the asymptotic behaviour of several types of moments of Borel probability measures on $\mathbf{R}^{d}$. In particular, we prove that the asymptotic behaviour of the moments of a measure is intimately related to the local dimensions of the measure.
\end{abstract}

\section{Introduction}

The purpose of this paper is to analyse the limiting behaviour of (different types of) moments of measures. In particular, we show that that there is a relationship between the asymptotic behaviour of the moments of a measure and the so-called local dimensions of the measure.

1.1. Local dimensions of measures. Let $\mu$ a Borel probability measure on $\mathbf{R}^{d}$. For $x \in \mathbf{R}^{d}$, we define the lower and upper local dimension of $\mu$ at $x$ by

$$
\underline{\operatorname{dim}}_{\mathrm{loc}}(\mu ; x)=\liminf _{r \searrow 0} \frac{\log \mu(B(x, r))}{\log r}
$$

and

$$
\overline{\operatorname{dim}}_{\text {loc }}(\mu ; x)=\limsup _{r \searrow 0} \frac{\log \mu(B(x, r))}{\log r},
$$

respectively. If the lower and upper local dimension of $\mu$ at $x$ coincide, then we write $\operatorname{dim}_{\text {loc }}(\mu ; x)$ for the common value, i.e. we write

$$
\operatorname{dim}_{\operatorname{loc}}(\mu ; x)=\lim _{r \searrow 0} \frac{\log \mu(B(x, r))}{\log r}
$$

provided the limit exists. The detailed study of the local dimensions of measures is known as multifractal analysis and has received enormous interest during the past 20 years; the reader is refereed to the texts [Fa2, $\mathrm{Pe}]$ for a more thorough discussion of this. It is now generally believed by experts that local dimensions provide important information about the geometric properties of measures.

1.2. Moments of measures. For a metric space $X$, we write

$$
\mathcal{P}(X)=\{\mu \mid \mu \text { is a Borel probability measure on } X\}
$$

and

$$
\mathcal{P}_{\text {co }}(X)=\{\mu \mid \mu \text { is a Borel probability measure on } X \text { with compact support }\} \text {. }
$$

In addition, we used the the following notation throughout the paper. Namely, if $\mu \in \mathcal{P}(X)$, then we will denote the support of $\mu$ by $\operatorname{supp} \mu$, and if $x \in X$, then we will denote the Dirac measure concentrated at $x$ by $\delta_{x}$. 
For $\mu \in \mathcal{P}_{\text {co }}\left(\mathbf{R}^{d}\right)$ and $q>0$, we define the $q^{\prime}$ th moment of $\mu$ by

$$
M_{q}(\mu)=\int|x|^{q} d \mu(x) .
$$

It is clear that if $\mu \in \mathcal{P}([0,1])$ satisfies $\mu(\{1\})=0$, then $M_{q}(\mu) \rightarrow 0$ as $q \rightarrow \infty$. It is therefore natural and of interest to ask for estimates of the rate at which $M_{q}(\mu)$ converges to 0 as $q \rightarrow \infty$, i.e. we ask for estimates of $\liminf _{q \rightarrow \infty} \frac{\log M_{q}(\mu)}{-\log q}$ and $\lim \sup _{q \rightarrow \infty} \frac{\log M_{q}(\mu)}{-\log q}$. For example, as an application of our main results we obtain the following result providing estimates of $\liminf \operatorname{in}_{q \rightarrow \infty} \frac{\log M_{q}(\mu)}{-\log q}$ and $\lim \sup _{q \rightarrow \infty} \frac{\log M_{q}(\mu)}{-\log q}$ in terms of the lower and upper local dimensions of $\mu$ at 1 .

Theorem 1.1. Let $\mu \in \mathcal{P}([0,1])$ with $0,1 \in \operatorname{supp} \mu$. Then

$$
\underline{\operatorname{dim}}_{\mathrm{loc}}(\mu ; 1) \leq \liminf _{q \rightarrow \infty} \frac{\log M_{q}(\mu)}{-\log q} \leq \limsup _{q \rightarrow \infty} \frac{\log M_{q}(\mu)}{-\log q} \leq \overline{\operatorname{dim}}_{\mathrm{loc}}(\mu ; 1) .
$$

In particular, if the local dimension $\operatorname{dim}_{\text {loc }}(\mu ; 1)$ exists then the $\operatorname{limit} \lim _{q \rightarrow \infty} \frac{\log M_{q}(\mu)}{-\log q}$ exists and

$$
\lim _{q \rightarrow \infty} \frac{\log M_{q}(\mu)}{-\log q}=\operatorname{dim}_{\mathrm{loc}}(\mu ; 1) .
$$

Proof. This follows from Corollary 2.8.

It is natural to consider other types of moments. In particular, in this paper, we will consider the following moment. For $\mu, \nu \in \mathcal{P}_{\text {co }}\left(\mathbf{R}^{d}\right)$ and $q>0$, we write

$$
M_{q}(\mu, \nu)=\int|x-y|^{q} d(\mu \times \nu)(x, y),
$$

i.e. $M_{q}(\mu, \nu)$ is the $q$ 'th moment of the average distance between two points $x$ and $y$ where $x$ is chosen with respect to the distribution $\mu$ and where $y$ is chosen with respect to the distribution $\nu$. As above, it is clear that if $\mu, \nu \in \mathcal{P}([0,1])$ satisfy $(\mu \times \nu)\left\{(x, y) \in[0,1]^{2}|| x-y \mid=1\right\}=0$ (i.e. if $(\mu \times \nu)\{(0,1)\}=0$ and $(\mu \times$ $\nu)\{(1,0)\}=0)$, then $M_{q}(\mu, \nu) \rightarrow 0$ as $q \rightarrow \infty$. It is therefore natural and of interest to ask for estimates of the rate at which $M_{q}(\mu, \nu)$ converges to 0 as $q \rightarrow \infty$, i.e. we ask for estimates of $\liminf \operatorname{in}_{q \rightarrow \infty} \frac{\log M_{q}(\mu, \nu)}{-\log q}$ and $\lim \sup _{q \rightarrow \infty} \frac{\log M_{q}(\mu, \nu)}{-\log q}$. As a further application of our main results we obtain the following result providing estimates of $\liminf _{q \rightarrow \infty} \frac{\log M_{q}(\mu, \nu)}{-\log q}$ and $\lim \sup _{q \rightarrow \infty} \frac{\log M_{q}(\mu, \nu)}{-\log q}$ in terms of the lower and upper local dimensions of $\mu$ and $\nu$ at 0 and at 1 .

Theorem 1.2. Let $\mu, \nu \in \mathcal{P}([0,1])$ with $0,1 \in \operatorname{supp} \mu$ and $0,1 \in \operatorname{supp} \nu$. Then

$$
\begin{aligned}
& \inf _{(x, y) \in\{(1,0),(0,1)\}}\left(\underline{\operatorname{dim}}_{\mathrm{loc}}(\mu ; x)+\underline{\operatorname{dim}}_{\mathrm{loc}}(\nu ; y)\right) \leq \liminf _{q \rightarrow \infty} \frac{\log M_{q}(\mu, \nu)}{-\log q} \\
& \leq \limsup _{q \rightarrow \infty} \frac{\log M_{q}(\mu, \nu)}{-\log q} \leq \inf _{(x, y) \in\{(1,0),(0,1)\}}\left(\overline{\operatorname{dim}}_{\mathrm{loc}}(\mu ; x)+\overline{\operatorname{dim}}_{\mathrm{loc}}(\nu ; y)\right) .
\end{aligned}
$$

In particular, if the local dimensions $\operatorname{dim}_{\text {loc }}(\mu ; 0), \operatorname{dim}_{\text {loc }}(\mu ; 1), \operatorname{dim}_{\text {loc }}(\nu ; 0)$ and $\operatorname{dim}_{\text {loc }}(\nu ; 1)$ exist, then the $\operatorname{limit}_{\lim _{q \rightarrow \infty}} \frac{\log M_{q}(\mu)}{-\log q}$ exists and

$$
\lim _{q \rightarrow \infty} \frac{\log M_{q}(\mu, \nu)}{-\log q}=\inf _{(x, y) \in\{(1,0),(0,1)\}}\left(\operatorname{dim}_{\mathrm{loc}}(\mu ; x)+\operatorname{dim}_{\mathrm{loc}}(\nu ; y)\right) .
$$

Proof. This follows from Corollary 2.7. 
We emphasise that Theorem 1.1 and Theorem 1.2 are special cases of the more general main results in Section 2. In particular, the results in Section 2 are not restricted to measures on $\mathbf{R}$ but apply to measures in all higher dimensional Euclidean spaces.

\section{Statements of results}

2.1. The extremal distance $d(\mu, \nu)$ and the extremal set $E(\mu, \nu)$. For $\mu, \nu \in \mathcal{P}_{\text {co }}\left(\mathbf{R}^{d}\right)$, we define the extremal distance $d(\mu, \nu)$ of $\mu$ and $\nu$ by

$$
d(\mu, \nu)=\sup _{\substack{x \in \operatorname{supp} \mu \\ y \in \operatorname{supp} \nu}}|x-y|
$$

and we define the extremal set $E(\mu, \nu)$ of $\mu$ and $\nu$ by

$$
E(\mu, \nu)=\{(x, y) \in \operatorname{supp} \mu \times \operatorname{supp} \nu|| x-y \mid=d(\mu, \nu)\} .
$$

It is clear that the extremal distance $d(\mu, \nu)$ is positive, i.e. $d(\mu, \nu) \geq 0$. It is also clear that if $d(\mu, \nu)=0$, then there is a point $\left(x_{0}, y_{0}\right) \in \mathbf{R}^{d} \times \mathbf{R}^{d}$, such that $\mu \times \nu=\delta_{\left(x_{0}, y_{0}\right)}$, whence $M_{q}(\mu, \nu)=\left|x_{0}-y_{0}\right|^{q}$ for all $q>1$, and so $\frac{\log M_{q}(\mu, \nu)}{-\log q} \rightarrow \infty$ if $\left|x_{0}-y_{0}\right|<1$ and $\frac{\log M_{q}(\mu, \nu)}{-\log q} \rightarrow-\infty$ if $\left|x_{0}-y_{0}\right|>1$. We may therefore assume that $d(\mu, \nu) \neq 0$. The next results show that if $d(\mu, \nu) \neq 1$, then the limiting behaviour of $\frac{\log M_{q}(\mu, \nu)}{-\log q}$ is also trivial.

Proposition 2.1. Let $\mu, \nu \in \mathcal{P}_{\text {co }}\left(\mathbf{R}^{d}\right)$.

(1) If $0<d(\mu, \nu)<1$, then

$$
\lim _{q \rightarrow \infty} \frac{\log M_{q}(\mu, \nu)}{-\log q}=\infty .
$$

(2) If $1<d(\mu, \nu)$, then

$$
\lim _{q \rightarrow \infty} \frac{\log M_{q}(\mu, \nu)}{-\log q}=-\infty .
$$

Proof. (1) If $0<d(\mu, \nu)<1$, then $M_{q}(\mu, \nu) \leq \int d(\mu, \nu)^{q} d(\mu \times \nu)(x, y)=d(\mu, \nu)^{q}$ for all $q>1$, and so $\frac{\log M_{q}(\mu, \nu)}{-\log q} \geq-\log (d(\mu, \nu)) \frac{q}{\log q} \rightarrow \infty$.

(2) Assume that $d(\mu, \nu)>1$ and choose $R$ such that $1<R<d(\mu, \nu)$. Now write

$$
G=\left\{(x, y) \in \mathbf{R}^{d} \times \mathbf{R}^{d}|| x-y \mid>R\right\} .
$$

It is clear that $G$ is open. Next, we show that

$$
G \cap \operatorname{supp}(\mu \times \nu) \neq \varnothing .
$$

We will now prove (2.3). Since $R<d(\mu, \nu)=\sup _{x \in \operatorname{supp} \mu, y \in \operatorname{supp} \nu}|x-y|$, we can find $x_{0} \in \operatorname{supp} \mu$ and $y_{0} \in \operatorname{supp} \nu$ such that $\left|x_{0}-y_{0}\right|>R$. This implies that $\left(x_{0}, y_{0}\right) \in G$ and that $\left(x_{0}, y_{0}\right) \in \operatorname{supp} \mu \times \operatorname{supp} \nu=\operatorname{supp}(\mu \times \nu)$, whence $\left(x_{0}, y_{0}\right) \in G \cap \operatorname{supp}(\mu \times \nu)$, and so $G \cap \operatorname{supp}(\mu \times \nu) \neq \varnothing$. This proves $(2.3)$.

Since $G$ is open and $G \cap \operatorname{supp}(\mu \times \nu) \neq \varnothing$ (by (2.3)), we conclude that $a=$ $(\mu \times \nu)(G)>0$. Next, we note that $M_{q}(\mu, \nu) \geq \int_{G}|x-y|^{q} d(\mu \times \nu)(x, y) \geq \int_{G} R^{q} d(\mu \times$ $\nu)(x, y)=a R^{q}$ for all $q>1$, whence $\frac{\log M_{q}(\mu, \nu)}{-\log q} \leq-\log (a) \frac{1}{\log q}-\log (R) \frac{q}{\log q} \rightarrow-\infty$ because $R>1$ and $a>0$. 
It follows from Proposition 2.1 and the preceding discussion, that if $d(\mu, \nu) \neq 1$, then the limiting behaviour of $\frac{\log M_{q}(\mu, \nu)}{-\log q}$ is trivial. Hence, below we will always assume that

$$
d(\mu, \nu)=1
$$

2.2. The main results. The first main result, i.e. Theorem 2.2 , provides an upper bound for $\lim \sup _{q \rightarrow \infty} \frac{\log M_{q}(\mu, \nu)}{-\log q}$ and our second main result, i.e. Theorem 2.4, provides a lower bound for $\liminf _{q \rightarrow \infty} \frac{\log M_{q}(\mu, \nu)}{-\log q}$. Below we use the following notation, namely, if $C$ is a subset $\mathbf{R}^{m}$, then we write $\operatorname{dim}_{\mathrm{H}} C$ for the Hausdorff dimension of $C$ and we write $\underline{\operatorname{dim}}_{\mathrm{B}} C$ and $\overline{\operatorname{dim}}_{\mathrm{B}} C$ for the lower and upper box dimension of $C$, respectively; the reader is referred to [Fa1] for the definition of the Hausdorff dimension and for the definitions of the lower and upper box dimensions. We can now state our first main result providing an upper bound for $\lim \sup _{q \rightarrow \infty} \frac{\log M_{q}(\mu, \nu)}{-\log q}$.

Theorem 2.2. Upper bound for $\limsup _{q \rightarrow \infty} \frac{\log M_{q}(\mu, \nu)}{-\log q}$. Let $\mu, \nu \in \mathcal{P}_{\text {co }}\left(\mathbf{R}^{d}\right)$ and assume that $d(\mu, \nu)=1$.

(1) We have

$$
\limsup _{q \rightarrow \infty} \frac{\log M_{q}(\mu, \nu)}{-\log q} \leq-\operatorname{dim}_{\mathrm{H}} E(\mu, \nu)+\sup _{(x, y) \in E(\mu, \nu)}\left(\overline{\operatorname{dim}}_{\mathrm{loc}}(\mu ; x)+\overline{\operatorname{dim}}_{\mathrm{loc}}(\nu ; y)\right)
$$

(2) We have

$$
\limsup _{q \rightarrow \infty} \frac{\log M_{q}(\mu, \nu)}{-\log q} \leq \inf _{(x, y) \in E(\mu, \nu)}\left(\overline{\operatorname{dim}}_{\mathrm{loc}}(\mu ; x)+\overline{\operatorname{dim}}_{\mathrm{loc}}(\nu ; y)\right) .
$$

The proof of Theorem 2.2 is given in Section 4 using the preliminary auxiliary results from Section 3,

In order to state our second main result providing a lower bound for $\lim \inf _{q \rightarrow \infty}$ $\frac{\log M_{q}(\mu, \nu)}{-\log q}$, we introduce the following covering condition. Below we use the following notation. If $C$ is a subset of $\mathbf{R}^{m}$ and $r>0$, then we write $B(C, r)$ for the $r$ neighbourhood of $C$, i.e.

$$
B(C, r)=\left\{x \in \mathbf{R}^{m} \mid \operatorname{dist}(x, C)<r\right\} .
$$

The extremal set covering condition. Let $\mu, \nu \in \mathcal{P}_{\text {со }}\left(\mathbf{R}^{d}\right)$ and assume that $d(\mu, \nu)=1$. For $0 \leq r \leq 1$, write

$$
E_{r}=\{(x, y) \in \operatorname{supp} \mu \times \operatorname{supp} \nu|| x-y \mid \geq 1-r\} .
$$

We will say that $\mu$ and $\nu$ satisfy the extremal set covering condition if there is a constant $k_{0}>0$ and a number $0<r_{0}<1$ such that

$$
E_{r} \subseteq B\left(E_{0}, k_{0} r\right)
$$

for all $0<r<r_{0}$.

Before presenting our second main result, i.e. Theorem 2.4, we first note that all measures $\mu$ and $\nu$ in $\mathbf{R}$ satisfy the extremal covering set condition; this is the content of Lemma 2.3 below.

Lemma 2.3. Let $\mu, \nu \in \mathcal{P}_{\text {со }}(\mathbf{R})$ and assume that $d(\mu, \nu)=1$. Then $\mu$ and $\nu$ satisfy the extremal set covering set condition. 
Proof. Since $\mu$ and $\nu$ have compact supports, we can find real numbers $s, t, u, v$ such that $\mu \in \mathcal{P}([s, t])$ with $s, t \in \operatorname{supp} \mu$ and $\nu \in \mathcal{P}([u, v])$ with $u, v \in \operatorname{supp} \nu$. Now write

$$
E= \begin{cases}\{(s, v)\} & \text { if }|s-v|>|u-t| ; \\ \{(s, v),(t, u)\} & \text { if }|s-v|=|u-t| ; \\ \{(t, u)\} & \text { if }|s-v|<|u-t|\end{cases}
$$

and note that $E=E(\mu, \nu)$. For $0 \leq r \leq 1$, let $E_{r}$ is defined as in (2.4). Since $E=E(\mu, \nu)=E_{0}$, it is easily seen that

$$
\begin{aligned}
E_{r} \subseteq\{ & (x, y) \in[s, t] \times[u, v]|| x-y \mid \geq 1-r\} \\
\subseteq & \left\{\begin{array}{l}
{[s, s+r] \times[v-r, v] \subseteq B(E, \sqrt{2} r)=B\left(E_{0}, \sqrt{2} r\right)} \\
\text { for } 0<r<1-|u-t| \text { if }|s-v|>|u-t| ; \\
([s, s+r] \times[v-r, r]) \cup([t-r, t] \times[u, u+r]) \subseteq B(E, \sqrt{2} r) \\
=B\left(E_{0}, \sqrt{2} r\right) \text { for } 0<r<1 \text { if }|s-v|=|u-t| ; \\
{[t-r, t] \times[u, u+r] \subseteq B(E, \sqrt{2} r)=B\left(E_{0}, \sqrt{2} r\right)} \\
\text { for } 0<r<1-|s-v| \text { if }|s-v|<|u-t| .
\end{array}\right.
\end{aligned}
$$

It follows immediately from (2.6) that $\mu$ and $\nu$ satisfy the extremal set covering condition.

Theorem 2.4. Lower bound for $\liminf _{q \rightarrow \infty} \frac{\log M_{q}(\mu, \nu)}{-\log q}$. Let $\mu, \nu \in \mathcal{P}_{\text {co }}\left(\mathbf{R}^{d}\right)$ and assume that $d(\mu, \nu)=1$. If $\mu$ and $\nu$ satisfy the extremal set covering condition, then we have

$$
\liminf _{q \rightarrow \infty} \frac{\log M_{q}(\mu, \nu)}{-\log q} \geq-\overline{\operatorname{dim}}_{\mathrm{B}} E(\mu, \nu)+\liminf _{r \searrow 0} \inf _{(x, y) \in E(\mu, \nu)} \frac{\log (\mu \times \nu) B((x, y), r)}{\log r} .
$$

The proof of Theorem 2.4 is given in Section 5 using the preliminary auxiliary results from Section 3.

While the second term in the upper bound for $\lim \sup _{q \rightarrow \infty} \frac{\log M_{q}(\mu, \nu)}{-\log q}$ in Theorem 2.2.(1) and the second term in the lower bound for $\liminf _{q \rightarrow \infty} \frac{\log M_{q}(\mu, \nu)}{-\log q}$ in Theorem 2.4 resemble each other, they represent two opposed set of ideas: the second term in upper bound for $\lim \sup _{q \rightarrow \infty} \frac{\log M_{q}(\mu, \nu)}{-\log q}$ in Theorem 2.2.(1), i.e. the term

$$
\begin{aligned}
& \sup _{(x, y) \in E(\mu, \nu)}\left(\overline{\operatorname{dim}}_{\text {loc }}(\mu ; x)+\overline{\operatorname{dim}}_{\text {loc }}(\nu ; y)\right) \\
& =\sup _{(x, y) \in E(\mu, \nu)}\left(\limsup _{r \searrow 0} \frac{\log \mu(B(x, r))}{\log r}+\limsup _{r \searrow 0} \frac{\log \nu(B(y, r))}{\log r}\right),
\end{aligned}
$$

is obtained by first letting $r$ tend to 0 (leading to the local dimensions $\overline{\operatorname{dim}}_{\text {loc }}(\mu ; x)$ $\left.\overline{\operatorname{dim}}_{\text {loc }}(\nu ; y)\right)$ followed by forming an extremum, whereas the corresponding term in lower bound for $\liminf _{q \rightarrow \infty} \frac{\log M_{q}(\mu, \nu)}{-\log q}$ in Theorem 2.4, i.e. the term

$$
\liminf _{r \searrow 0} \inf _{(x, y) \in E(\mu, \nu)} \frac{\log (\mu \times \nu) B((x, y), r)}{\log r},
$$

is obtained by first forming an extremum (the infimum $\inf _{(x, y) \in E(\mu, \nu)} \frac{\log (\mu \times \nu) B((x, y), r)}{\log r}$ ) followed by letting $r$ tend to 0 . However, under an additional condition, namely Condition (2.9) below, the term (2.8) can be brought into a form that is similar to (2.7); this is the statement of Corollary 2.5 below. In order to state Condition (2.9) 
in Corollary 2.5, we introduce the following notation. For a probability measure $\gamma$ on $\mathbf{R}^{m}$, we write

$$
\underline{\operatorname{dim}}_{\mathrm{loc}}(\gamma ; x ; r)=\inf _{0<\rho \leq r} \frac{\log \gamma(B(x, \rho))}{\log \rho} .
$$

It follows immediately from the definition of $\underline{\operatorname{dim}}_{\mathrm{loc}}(\gamma ; x ; r)$ and $\underline{\operatorname{dim}}_{\mathrm{loc}}(\gamma ; x)$ that

$$
\underline{\operatorname{dim}}_{\mathrm{loc}}(\gamma ; x ; r) \nearrow \underline{\operatorname{dim}}_{\mathrm{loc}}(\gamma ; x) \text { for all } x \in \mathbf{R}^{m} \text { as } r \searrow 0 \text {. }
$$

In particular, we conclude that if $\mu, \nu \in \mathcal{P}_{\text {co }}\left(\mathbf{R}^{d}\right)$, then

$$
\underline{\operatorname{dim}}_{\mathrm{loc}}(\mu \times \nu ;(x, y) ; r) \nearrow \underline{\operatorname{dim}}_{\mathrm{loc}}(\mu \times \nu ;(x, y)) \quad \text { for all }(x, y) \in \mathbf{R}^{d} \times \mathbf{R}^{d} \text { as } r \searrow 0 \text {. }
$$

While simple examples show that $\underline{\operatorname{dim}}_{\text {loc }}(\mu \times \nu ; \cdot ; r)$ does not necessarily convergence uniformly to $\underline{\operatorname{dim}}_{\mathrm{loc}}(\mu \times \nu ; \cdot)$ as $r \searrow 0$, Condition $(2.9)$ in Corollary 2.5 below stipulates that $\underline{\operatorname{dim}}_{\mathrm{loc}}(\mu \times \nu ; \cdot ; r)$ converges uniformly to $\underline{\operatorname{dim}}_{\mathrm{loc}}(\mu \times \nu ; \cdot)$ on $E(\mu, \nu)$ as $r \searrow 0$. We can now state Corollary 2.5.

Corollary 2.5. Lower bound for $\liminf _{q \rightarrow \infty} \frac{\log M_{q}(\mu, \nu)}{-\log q}$. Let $\mu, \nu \in \mathcal{P}_{\text {co }}\left(\mathbf{R}^{d}\right)$ and assume that $d(\mu, \nu)=1$. If $\mu$ and $\nu$ satisfy the extremal set covering condition and

$$
\underline{\operatorname{dim}}_{\mathrm{loc}}(\mu \times \nu ; \cdot ; r) \rightarrow \underline{\operatorname{dim}}_{\mathrm{loc}}(\mu \times \nu ; \cdot) \quad \text { uniformly on } E(\mu, \nu) \text { as } r \searrow 0 \text {, }
$$

then

$$
\liminf _{q \rightarrow \infty} \frac{\log M_{q}(\mu, \nu)}{-\log q} \geq-\overline{\operatorname{dim}}_{\mathrm{B}} E(\mu, \nu)+\inf _{(x, y) \in E(\mu, \nu)}\left(\underline{\operatorname{dim}}_{\mathrm{loc}}(\mu ; x)+\underline{\operatorname{dim}}_{\mathrm{loc}}(\nu ; y)\right) .
$$

Proof. It is not difficult to see that (2.9) implies that

$$
\text { (2.10) } \lim _{r \searrow 0} \inf _{(x, y) \in E(\mu, \nu)} \underline{\operatorname{dim}}_{\mathrm{loc}}(\mu \times \nu ;(x, y) ; r)=\inf _{(x, y) \in E(\mu, \nu)} \lim _{r \searrow 0} \underline{\operatorname{dim}}_{\mathrm{loc}}(\mu \times \nu ;(x, y) ; r) \text {. }
$$

Using the fact that $\underline{\operatorname{dim}}_{\mathrm{loc}}(\mu \times \nu ;(x, y) ; r)=\inf _{0<\rho \leq r} \frac{\log (\mu \times \nu) B((x, y), \rho)}{\log \rho}$ combined with the fact that $\lim _{r \searrow 0} \underline{\operatorname{dim}}_{\text {loc }}(\mu \times \nu ;(x, y) ; r)=\liminf _{r \searrow 0} \frac{\log (\mu(B(x, r)) \nu(B(y, r)))}{\log r},(2.10)$ can now be rewritten as

$$
\begin{aligned}
& \lim _{r \searrow 0} \inf _{(x, y) \in E(\mu, \nu)} \inf _{0<\rho \leq r} \frac{\log (\mu \times \nu) B((x, y), \rho)}{\log \rho} \\
& =\inf _{(x, y) \in E(\mu, \nu)} \liminf _{r \searrow 0} \frac{\log (\mu(B(x, r)) \nu(B(y, r)))}{\log r} .
\end{aligned}
$$

Next, since clearly

$$
\inf _{0<\rho \leq r} \inf _{(x, y) \in E(\mu, \nu)} \frac{\log (\mu \times \nu) B((x, y), \rho)}{\log \rho}=\inf _{(x, y) \in E(\mu, \nu)} \inf _{0<\rho \leq r} \frac{\log (\mu \times \nu) B((x, y), \rho)}{\log \rho},
$$

we now deduce from the previous equality that

$$
\begin{aligned}
& \liminf _{r \searrow 0} \inf _{(x, y) \in E(\mu, \nu)} \frac{\log (\mu \times \nu) B((x, y), r)}{\log r} \\
& =\lim _{r \searrow 0} \inf _{0<\rho \leq r} \inf _{(x, y) \in E(\mu, \nu)} \frac{\log (\mu \times \nu) B((x, y), \rho)}{\log \rho} \\
& =\lim _{r \searrow 0} \inf _{(x, y) \in E(\mu, \nu)} \inf _{0<\rho \leq r} \frac{\log (\mu \times \nu) B((x, y), \rho)}{\log \rho} \\
& =\inf _{(x, y) \in E(\mu, \nu)} \liminf _{r \searrow 0} \frac{\log (\mu(B(x, r)) \nu(B(y, r)))}{\log r} .
\end{aligned}
$$


However, since $B((x, y), r) \subseteq B(x, r) \times B(y, r)$ for all $(x, y) \in \mathbf{R}^{d} \times \mathbf{R}^{d}$ and all $r>0$, we conclude from (2.11) that

$$
\begin{aligned}
& \liminf _{r \searrow 0} \inf _{(x, y) \in E(\mu, \nu)} \frac{\log (\mu \times \nu) B((x, y), r)}{\log r} \\
& \geq \inf _{(x, y) \in E(\mu, \nu)} \liminf _{r \searrow 0} \frac{\log (\mu(B(x, r)) \nu(B(y, r)))}{\log r} \\
& =\inf _{(x, y) \in E(\mu, \nu)} \liminf _{r \searrow 0}\left(\frac{\log \mu(B(x, r)}{\log r}+\frac{\log \nu(B(y, r)}{\log r}\right) \\
& \geq \inf _{(x, y) \in E(\mu, \nu)}\left(\liminf _{r \searrow 0} \frac{\log \mu(B(x, r)}{\log r}+\liminf _{r \searrow 0} \frac{\log \nu(B(y, r)}{\log r}\right) \\
& =\inf _{(x, y) \in E(\mu, \nu)}\left(\underline{\operatorname{dim}}_{\operatorname{loc}}(\mu ; x)+\underline{\operatorname{dim}}_{\operatorname{loc}}(\nu ; y)\right) .
\end{aligned}
$$

The desired statement now follows from (2.12) and Theorem 2.4.

Before presenting several applications of Theorem 2.2 and Theorem 2.4, we first made the following two comments.

Remark 1. For $\mu, \nu \in \mathcal{P}_{\text {co }}\left(\mathbf{R}^{d}\right)$, write

$$
\triangleq(\mu, \nu)=\inf _{(x, y) \in E(\mu, \nu)}\left(\overline{\operatorname{dim}}_{\mathrm{loc}}(\mu ; x)+\overline{\operatorname{dim}}_{\mathrm{loc}}(\nu ; y)\right)
$$

and

$$
\bar{\Delta}(\mu, \nu)=\sup _{(x, y) \in E(\mu, \nu)}\left(\overline{\operatorname{dim}}_{\mathrm{loc}}(\mu ; x)+\overline{\operatorname{dim}}_{\mathrm{loc}}(\nu ; y)\right) .
$$

Using this notation, Theorem 2.2 says that if $d(\mu, \nu)=1$, then the numbers $-\operatorname{dim}_{\mathrm{H}} E(\mu, \nu)+\bar{\Delta}(\mu, \nu)$ and $\underline{\Delta}(\mu, \nu)$ are upper bounds for $\lim \sup _{q \rightarrow \infty} \frac{\log M_{q}(\mu, \nu)}{-\log q}$, i.e.

$$
\limsup _{q \rightarrow \infty} \frac{\log M_{q}(\mu, \nu)}{-\log q} \leq-\operatorname{dim}_{\mathrm{H}} E(\mu, \nu)+\bar{\Delta}(\mu, \nu)
$$

and

$$
\limsup _{q \rightarrow \infty} \frac{\log M_{q}(\mu, \nu)}{-\log q} \leq \triangleq(\mu, \nu)
$$

We will now provide examples showing that, in general, there is no relation between the upper bounds $-\operatorname{dim}_{\mathrm{H}} E(\mu, \nu)+\bar{\Delta}(\mu, \nu)$ and $\underline{\Delta}(\mu, \nu)$. More precisely, we will construct measures $\mu_{p}, \nu \in \mathcal{P}_{\text {co }}\left(\mathbf{R}^{2}\right)$ for $0<p \leq \frac{1}{2}$ such that $-\operatorname{dim}_{\mathrm{H}} E\left(\mu_{p}, \nu\right)+$ $\bar{\Delta}\left(\mu_{p}, \nu\right)>\triangleq\left(\mu_{p}, \nu\right)$ for $0<p<\frac{1}{3},-\operatorname{dim}_{\mathrm{H}} E\left(\mu_{p}, \nu\right)+\bar{\Delta}\left(\mu_{p}, \nu\right)=\Delta\left(\mu_{p}, \nu\right)$ for $p=\frac{1}{3}$ and $-\operatorname{dim}_{\mathrm{H}} E\left(\mu_{p}, \nu\right)+\bar{\Delta}\left(\mu_{p}, \nu\right)<\underline{\Delta}\left(\mu_{p}, \nu\right)$ for $\frac{1}{3}<p \leq \frac{1}{2}$. Fix $0<r<\frac{1}{2}$ and define $S_{1}, S_{2}:[0,1] \rightarrow[0,1]$ by $S_{1}(x)=r x$ and $S_{2}(x)=r x+1-r$. Let $C$ be the self-similar set associated with the list $\left(S_{i}\right)_{i}$, i.e. $C$ is the unique non-empty compact subset of $[0,1]$ such that $C=\cup_{i} S_{i}(C)$. Also, fix $0<p \leq \frac{1}{2}$ and consider the probability vector $\left(p_{1}, p_{2}\right)=(p, 1-p)$. Next, let $\pi_{p}$ be the self-similar measure associated with the list $\left(S_{i}, p_{i}\right)_{i}$, i.e. $\pi_{p}$ is the unique Borel probability measure on $C$ such that $\pi_{p}=\sum_{i} p_{i} \pi_{p} \circ S_{i}^{-1}$. We now define $\varphi:[0,1] \rightarrow \mathbf{R}^{2}$ by $\varphi(t)=(\cos (2 \pi t), \sin (2 \pi t))$. Finally, we define $\mu_{p}, \nu \in \mathcal{P}_{\text {co }}\left(\mathbf{R}^{2}\right)$ by $\mu_{p}=\pi_{p} \circ \varphi^{-1}$ and $\nu=\delta_{(0,0)}$. Since $\operatorname{supp} \pi_{p}=C$, it is clear that $\operatorname{supp} \mu_{p}=\varphi(C)$. It is also clear that $\operatorname{supp} \nu=\{(0,0)\}$ and $d\left(\mu_{p}, \nu\right)=1$. The previous remarks imply that

$$
E\left(\mu_{p}, \nu\right)=\operatorname{supp}\left(\mu_{p} \times \nu\right)=\operatorname{supp} \mu_{p} \times \operatorname{supp} \nu=\varphi(C) \times\{(0,0)\} .
$$


Since $\operatorname{dim}_{\mathrm{H}} \varphi(C)=\operatorname{dim}_{\mathrm{H}} C$ (because $\varphi$ is bi-Lipschitz) and $\operatorname{dim}_{\mathrm{H}} C=-\frac{\log 2}{\log r}$ (see [Fa1]), we conclude from (2.13) that

$$
\operatorname{dim}_{\mathrm{H}} E\left(\mu_{p}, \nu\right)=\operatorname{dim}_{\mathrm{H}} \varphi(C)=\operatorname{dim}_{\mathrm{H}} C=-\frac{\log 2}{\log r} .
$$

Next, we note that it is well-known that $\sup _{t \in C} \overline{\operatorname{dim}}_{\text {loc }}\left(\pi_{p} ; t\right)=\max _{i} \frac{\log p_{i}}{\log r}=\frac{\log p}{\log r}$ and $\inf _{t \in C} \overline{\operatorname{dim}}_{\operatorname{loc}}\left(\pi_{p} ; t\right)=\min _{i} \frac{\log p_{i}}{\log r}=\frac{\log (1-p)}{\log r}$, see [CaMa] or [Fa2]. Since it is also not difficult to see that $\overline{\operatorname{dim}}_{\text {loc }}\left(\pi_{p} ; t\right)=\overline{\operatorname{dim}}_{\text {loc }}\left(\mu_{p} ; \varphi(t)\right)$ for all $t \in C$, we therefore conclude that

$$
\begin{aligned}
\Delta\left(\mu_{p}, \nu\right) & =\inf _{(x, y) \in E\left(\mu_{p}, \nu\right)}\left(\overline{\operatorname{dim}}_{\mathrm{loc}}\left(\mu_{p} ; x\right)+\overline{\operatorname{dim}}_{\mathrm{loc}}(\nu ; y)\right) \\
& =\inf _{x \in \varphi(C)} \overline{\operatorname{dim}}_{\mathrm{loc}}\left(\mu_{p} ; x\right)=\inf _{t \in C} \overline{\operatorname{dim}}_{\mathrm{loc}}\left(\pi_{p} ; t\right)=\frac{\log (1-p)}{\log r} .
\end{aligned}
$$

Similarly, we prove that $\bar{\Delta}\left(\mu_{p}, \nu\right)=\frac{\log p}{\log r}$. Combining this and (2.14) gives

$$
-\operatorname{dim}_{\mathrm{H}} E\left(\mu_{p}, \nu\right)+\bar{\Delta}\left(\mu_{p}, \nu\right)=\frac{\log 2}{\log r}+\frac{\log p}{\log r}=\frac{\log 2 p}{\log r} .
$$

It follows immediately from (2.15) and (2.16) that

$$
\begin{array}{ll}
-\operatorname{dim}_{\mathrm{H}} E\left(\mu_{p}, \nu\right)+\bar{\Delta}\left(\mu_{p}, \nu\right)>\Delta\left(\mu_{p}, \nu\right) & \text { for } 0<p<\frac{1}{3} \\
-\operatorname{dim}_{\mathrm{H}} E\left(\mu_{p}, \nu\right)+\bar{\Delta}\left(\mu_{p}, \nu\right)=\triangleq\left(\mu_{p}, \nu\right) & \text { for } p=\frac{1}{3} \\
-\operatorname{dim}_{\mathrm{H}} E\left(\mu_{p}, \nu\right)+\bar{\Delta}\left(\mu_{p}, \nu\right)<\triangleq\left(\mu_{p}, \nu\right) & \text { for } \frac{1}{3}<p \leq \frac{1}{2} .
\end{array}
$$

This concludes the first remark.

Remark 2. The second term in the lower bound for $\liminf _{q \rightarrow \infty} \frac{\log M_{q}(\mu, \nu)}{-\log q}$ in Theorem 2.4, i.e. the term

$$
\begin{aligned}
& \liminf _{r \searrow 0} \inf _{(x, y) \in E(\mu, \nu)} \frac{\log (\mu \times \nu) B((x, y), r)}{\log r} \\
& =\liminf _{r \searrow 0} \frac{\log \sup _{(x, y) \in E(\mu, \nu)}(\mu \times \nu) B((x, y), r)}{\log r},
\end{aligned}
$$

is closely related to the so-called lower $\infty$-Rényi dimension of $\mu \times \nu$ and the concentration function of $\mu \times \nu$. We will now explain these connections. For a Borel probability measure $\gamma$ on $\mathbf{R}^{m}$ and $q \in[-\infty, \infty]$, we define the lower $q$-Rényi dimensions of $\gamma$ by

$$
\begin{aligned}
& \underline{D}_{\gamma}(q)=\liminf _{r \searrow 0} \frac{1}{q-1} \frac{\log \int_{\operatorname{supp} \gamma} \gamma(B(x, r))^{q-1} d \gamma(x)}{\log r} \text { for } q \in \mathbf{R} \backslash\{1\}, \\
& \underline{D}_{\gamma}(1)=\liminf _{r \searrow 0} \frac{\int_{\operatorname{supp} \gamma} \log \gamma(B(x, r)) d \gamma(x)}{\log r},
\end{aligned}
$$

and

$$
\begin{aligned}
\underline{D}_{\gamma}(-\infty) & =\liminf _{r \searrow 0} \frac{\log \inf _{x \in \operatorname{supp} \gamma} \gamma(B(x, r))}{\log r}, \\
\underline{D}_{\gamma}(\infty) & =\liminf _{r \searrow 0} \frac{\log \sup _{x \in \operatorname{supp} \gamma} \gamma(B(x, r))}{\log r} .
\end{aligned}
$$

The upper Rényi dimensions are defined analogously by replacing lim inf by limsup. It is clear that if $E(\mu, \nu)=\operatorname{supp}(\mu \times \nu)$ (and this may happen, see, for example, 
(2.13)), then the second term in the lower bound for $\liminf \inf _{q \rightarrow \infty} \frac{\log M_{q}(\mu, \nu)}{-\log q}$ in Theorem 2.4, equals lower $\infty$-Rényi dimension of $\mu \times \nu$, i.e.

$$
\begin{aligned}
\liminf _{r \searrow 0} \inf _{(x, y) \in E(\mu, \nu)} \frac{\log (\mu \times \nu) B((x, y), r)}{\log r} & =\liminf _{r \searrow 0} \frac{\log \sup _{(x, y) \in E(\mu, \nu)}(\mu \times \nu) B((x, y), r)}{\log r} \\
& =\underline{D}_{\mu \times \nu}(\infty) .
\end{aligned}
$$

The Rényi dimensions were essentially introduced by Rényi [Ré1, Ré2] in 1960 as a tool for analyzing various problems in information theory. The main significance of the Rényi dimensions, is their relationship with the multifractal spectrum of the measure $\gamma$. We define the Hausdorff multifractal spectrum function, $f_{\gamma}$, of $\gamma$ by

$$
f_{\gamma}(\alpha)=\operatorname{dim}_{\mathrm{H}}\left\{\begin{array}{l|l}
x \in \mathbf{R}^{m} & \lim _{r \searrow 0} \frac{\log \gamma(B(x, r))}{\log r}=\alpha
\end{array}\right\}, \quad \alpha \geq 0 .
$$

In the 1980's it was conjectured in the physics literature [HaJeKaPrSh] that for "good" measures the following result, relating the multifractal spectrum function $f_{\gamma}$ to the Legendre transform of the Rényi dimensions, holds: the multifractal spectrum function $f_{\gamma}$ coincides with the Legendre transforms of the functions $\underline{\tau}_{\gamma}, \bar{\tau}_{\gamma}: \mathbf{R} \rightarrow \mathbf{R}$ define by $\underline{\tau}_{\gamma}(q)=(1-q) \underline{D}_{\gamma}(q)$ and $\underline{\tau}_{\gamma}(q)=(1-q) \bar{D}_{\gamma}(q)$, i.e. $f_{\gamma}(\alpha)=\underline{\tau}_{\gamma}^{*}(\alpha)=$ $\bar{\tau}_{\gamma}^{*}(\alpha)$ for all $\alpha \geq 0$ where $\underline{\tau}_{\gamma}^{*}$ and $\bar{\tau}_{\gamma}^{*}$ denote the Legendre transforms of $\underline{\tau}_{\gamma}$ and $\bar{\tau}_{\gamma}$, respectively. This result is known as the Multifractal Formalism. During the 1990's there has been an enormous interest in verifying the Multifractal Formalism and computing the multifractal spectra of measures in the mathematical literature, see $[\mathrm{Fa} 2, \mathrm{Pe}]$.

The term in (2.17) is also related to concentration functions. If $P$ is a Borel probability measure on $\mathbf{R}^{m}$, then the concentration function $Q_{P}:(0, \infty) \rightarrow \mathbf{R}$ of $P$ is defined by $Q_{P}(r)=\sup _{x \in \mathbf{R}^{m}} P(B(x, r))$, see [HeTh]. Using this notation and terminology, it is now clear that if $E(\mu, \nu)=\operatorname{supp}(\mu \times \nu)$, then the term in (2.17) equals $\lim \inf _{r \searrow 0} \frac{\log Q_{\mu \times \nu}(r)}{\log r}$. This completes the second remark.

2.3. Applications of the main results. We now present several corollaries of Theorem 2.2, Theorem 2.4 and Corollary 2.5. In particular, we consider the following two special cases: (1) the extremal set $E(\mu, \nu)$ is finite, and (2) the measures $\mu$ and $\nu$ are measures on the real line $\mathbf{R}$.

Corollary 2.6. Let $\mu, \nu \in \mathcal{P}_{\text {co }}\left(\mathbf{R}^{d}\right)$ and assume that $d(\mu, \nu)=1$. If $\mu$ and $\nu$ satisfy the extremal set covering condition and $E(\mu, \nu)$ is finite, then

$$
\begin{aligned}
\inf _{(x, y) \in E(\mu, \nu)}\left(\underline{\operatorname{dim}}_{\mathrm{loc}}(\mu ; x)+\underline{\operatorname{dim}}_{\mathrm{loc}}(\nu ; y)\right) & \leq \liminf _{q \rightarrow \infty} \frac{\log M_{q}(\mu, \nu)}{-\log q} \leq \limsup _{q \rightarrow \infty} \frac{\log M_{q}(\mu, \nu)}{-\log q} \\
& \leq \inf _{(x, y) \in E(\mu, \nu)}\left(\overline{\operatorname{dim}}_{\mathrm{loc}}(\mu ; x)+\overline{\operatorname{dim}}_{\mathrm{loc}}(\nu ; y)\right) .
\end{aligned}
$$

Proof. Since $E(\mu, \nu)$ is finite we conclude that Condition (2.9) is satisfied and that $\operatorname{dim}_{\mathrm{H}} E(\mu, \nu)=\overline{\operatorname{dim}}_{\mathrm{B}} E(\mu, \nu)=0$. The desired result follows immediately from this and Theorem 2.2 and Corollary 2.5.

Corollary 2.7. Let $\mu \in \mathcal{P}([s, t])$ and $\nu \in \mathcal{P}([u, v])$ with $s, t \in \operatorname{supp} \mu$ and $u, v \in \operatorname{supp} \nu$ and assume that

$$
\max (|s-v|,|u-t|)=1 .
$$


Write

Then

$$
E= \begin{cases}\{(s, v)\} & \text { if }|s-v|>|u-t| \\ \{(s, v),(t, u)\} & \text { if }|s-v|=|u-t| \\ \{(t, u)\} & \text { if }|s-v|<|u-t|\end{cases}
$$

$$
\begin{aligned}
\inf _{(x, y) \in E}\left(\underline{\operatorname{dim}}_{\mathrm{loc}}(\mu ; x)+\underline{\operatorname{dim}}_{\mathrm{loc}}(\nu ; y)\right) & \leq \liminf _{q \rightarrow \infty} \frac{\log M_{q}(\mu, \nu)}{-\log q} \leq \limsup _{q \rightarrow \infty} \frac{\log M_{q}(\mu, \nu)}{-\log q} \\
& \leq \inf _{(x, y) \in E}\left(\overline{\operatorname{dim}}_{\mathrm{loc}}(\mu ; x)+\overline{\operatorname{dim}}_{\mathrm{loc}}(\nu ; y)\right) .
\end{aligned}
$$

Proof. It is clear that $d(\mu, \nu)=1$ (since $\max (|s-v|,|u-t|)=1$ ) and that $E(\mu, \nu)=E$. Next, we observe that $\mu$ and $\nu$ satisfy the extremal covering condition (by Lemma 2.3) and that $E(\mu, \nu)$ is finite (since $E(\mu, \nu)=E$ ). The desired result follows immediately from this observation and Corollary 2.6.

Recall, that for $\mu \in \mathcal{P}_{\text {co }}\left(\mathbf{R}^{d}\right)$ and $q>0$, we write

$$
M_{q}(\mu)=\int|x|^{q} d \mu(x) .
$$

Our final corollary provides estimates for $\lim \inf _{q \rightarrow \infty} \frac{\log M_{q}(\mu)}{-\log q}$ and $\lim \sup _{q \rightarrow \infty} \frac{\log M_{q}(\mu)}{-\log q}$ for $\mu \in \mathcal{P}([0,1])$.

Corollary 2.8. Let $\mu \in \mathcal{P}([0,1])$ with $1 \in \operatorname{supp} \mu$. Then

$$
\underline{\operatorname{dim}}_{\mathrm{loc}}(\mu ; 1) \leq \liminf _{q \rightarrow \infty} \frac{\log M_{q}(\mu)}{-\log q} \leq \limsup _{q \rightarrow \infty} \frac{\log M_{q}(\mu)}{-\log q} \leq \overline{\operatorname{dim}}_{\mathrm{loc}}(\mu ; 1) .
$$

Proof. This corollary follows from applying Corollary 2.7 to the measures $\mu$ and $\nu=\delta_{0}$ noticing that $M_{q}\left(\mu, \delta_{0}\right)=M_{q}(\mu)$.

\section{Proofs of Theorem 2.2 and Theorem 2.4. Preliminary results}

The purpose of this section is to provide various auxiliary results that will be used in the proofs of Theorem 2.2 and Theorem 2.4. The two main results results are Lemma 3.2 and Lemma 3.4. Lemma 3.2 provides an alternative expressing for the moments $M_{q}(\mu, \nu)$. This expression will (see Section 4 and Section 5) allow us to bound $M_{q}(\mu, \nu)$ by an integral of the form $\int_{1-\delta}^{1} q u^{q}(1-u)^{a} d u$ for suitable choices of $\delta$ and $a$, and Lemma 3.4 establishes the asymptotic behaviour of the integral $\int_{1-\delta}^{1} q u^{q}(1-u)^{a} d u$ as $q \rightarrow \infty$.

Before stating and proving the first main auxiliary result, namely Lemma 3.2, we first recall the following well-known result from analysis.

Lemma 3.1. Let $X$ be a separable metric space and let $m$ be a Borel measure on $X$. If $f: X \rightarrow[0, \infty)$ is a positive Borel function, then

$$
\int f d m=\int_{0}^{\infty} m(\{f \geq t\}) d t
$$

Proof. This result is proven in [Ma, Theorem 1.15].

Lemma 3.2. Let $\mu, \nu \in \mathcal{P}_{\text {со }}\left(\mathbf{R}^{d}\right)$ and assume that

$$
\sup _{\substack{x \in \operatorname{supp} \mu \\ y \in \operatorname{supp} \nu}}|x-y|=1
$$


Fix $0<\delta<1$. Then there is a function $h:(0, \infty) \rightarrow \mathbf{R}$ such that

$$
M_{q}(\mu, \nu)=\int_{1-\delta}^{1} q u^{q-1}(\mu \times \nu)\left\{(x, y) \in \mathbf{R}^{d} \times \mathbf{R}^{d}|| x-y \mid \geq u\right\} d u+h(q)
$$

and $|h(q)| \leq(1-\delta)^{q}$ for all $q>0$.

Proof. Define $f: \mathbf{R}^{d} \times \mathbf{R}^{d} \rightarrow[0, \infty)$ by $f(x, y)=|x-y|^{q}$. It now follows from Lemma 3.1 that

$$
\begin{aligned}
M_{q}(\mu, \nu) & =\int f d(\mu \times \nu)=\int_{0}^{\infty}(\mu \times \nu)(\{f \geq t\}) d t \\
& =\int_{0}^{\infty}(\mu \times \nu)\left\{(x, y) \in \mathbf{R}^{d} \times \mathbf{R}^{d}|| x-\left.y\right|^{q} \geq t\right\} d t \\
& =\int_{0}^{\infty}(\mu \times \nu)\left\{(x, y) \in \mathbf{R}^{d} \times \mathbf{R}^{d}|| x-y \mid \geq t^{\frac{1}{q}}\right\} d t .
\end{aligned}
$$

Introducing the substitution $u=t^{\frac{1}{q}}$ into the integral in (3.1), it now follows that

$$
M_{q}(\mu, \nu)=\int_{0}^{\infty} q u^{q-1}(\mu \times \nu)\left\{(x, y) \in \mathbf{R}^{d} \times \mathbf{R}^{d}|| x-y \mid \geq u\right\} d u
$$

and the assumption $\sup _{x \in \operatorname{supp} \mu, y \in \operatorname{supp} \nu}|x-y|=1$, therefore implies that

$$
M_{q}(\mu, \nu)=\int_{0}^{1} q u^{q-1}(\mu \times \nu)\left\{(x, y) \in \mathbf{R}^{d} \times \mathbf{R}^{d}|| x-y \mid \geq u\right\} d u .
$$

It follows immediately from (3.2) that

$$
M_{q}(\mu, \nu)=\int_{1-\delta}^{1} q u^{q-1}(\mu \times \nu)\left\{(x, y) \in \mathbf{R}^{d} \times \mathbf{R}^{d}|| x-y \mid \geq u\right\} d u+h(q),
$$

where $h(q)=\int_{0}^{1-\delta} q u^{q-1}(\mu \times \nu)\left\{(x, y) \in \mathbf{R}^{d} \times \mathbf{R}^{d}|| x-y \mid \geq u\right\} d u$. In particular, we conclude that $|h(q)| \leq \int_{0}^{1-\delta} q u^{q-1} d u=(1-\delta)^{q}$ for all $q>0$.

Next, we will state and prove the second main auxiliary result in this section, namely, Lemma 3.4. However, we first prove lemma 3.3 below.

Lemma 3.3. Fix $0<\delta<1$ and $a>0$. Then there are functions $f, g:(0, \infty) \rightarrow$ $\mathbf{R}$ and a real number $c$ such that

$$
\int_{1-\delta}^{1} q u^{q-1}(1-u)^{a} d u=c f(q) q^{-a}+g(q)
$$

and $f(q) \rightarrow 1$ as $q \rightarrow \infty$ and $|g(q)| \leq(1-\delta)^{q}$ for all $q>0$.

Proof. Define the function $f:(0, \infty) \rightarrow \mathbf{R}$ by $f(q)=q^{a} \frac{\Gamma(q+1)}{\Gamma(q+a+1)}$ and note that it follows from [Ol, p. 119] that $f(q) \rightarrow 1$ as $q \rightarrow \infty$. Also, define the real number $c$ by $c=\Gamma(a+1)$,

Next, define the function $g:(0, \infty) \rightarrow \mathbf{R}$ by $g(q)=-\int_{0}^{1-\delta} q u^{q-1}(1-u)^{a} d u$, and note that $|g(q)| \leq \int_{0}^{1-\delta} q u^{q-1}(1-u)^{a} d u \leq \int_{0}^{1-\delta} q u^{q-1} d u=(1-\delta)^{q}$ for all $q>0$. 
Finally, since it is easily seen that $\int_{0}^{1} u^{q-1}(1-u)^{a} d u=\frac{\Gamma(q) \Gamma(a+1)}{\Gamma(q+a+1)}$, we have

$$
\begin{aligned}
\int_{1-\delta}^{1} q u^{q-1}(1-u)^{a} d u & =\int_{0}^{1} q u^{q-1}(1-u)^{a} d u-\int_{0}^{1-\delta} q u^{q-1}(1-u)^{a} d u \\
& =q \frac{\Gamma(q) \Gamma(a+1)}{\Gamma(q+a+1)}+g(q)=\frac{\Gamma(q+1) \Gamma(a+1)}{\Gamma(q+a+1)}+g(q) \\
& =c f(q) q^{-a}+g(q)
\end{aligned}
$$

for all $q>0$.

Lemma 3.4. Fix $0<\delta<1, a>0$ and $m>0$. Let $h:(0, \infty) \rightarrow \mathbf{R}$ be a function and assume that $|h(q)| \leq(1-\delta)^{q}$ for all $q>0$. Then

$$
\lim _{q \rightarrow \infty} \frac{\log \left(m \int_{1-\delta}^{1} q u^{q-1}(1-u)^{a} d u+h(q)\right)}{-\log q}=a .
$$

Proof. It follows from Lemma 3.3 there are functions $f, g:(0, \infty) \rightarrow \mathbf{R}$ and a real number $c$ such that

$$
\int_{1-\delta}^{1} q u^{q-1}(1-u)^{a} d u=c f(q) q^{-a}+g(q)
$$

and $f(q) \rightarrow 1$ as $q \rightarrow \infty$ and $|g(q)| \leq(1-\delta)^{q}$ for all $q>0$. In particular, this shows that

$$
m \int_{1-\delta}^{1} q u^{q-1}(1-u)^{a} d u+h(q)=m c f(q) q^{-a}+m g(q)+h(q)=q^{-a} \varphi(q)
$$

where the function $\varphi:(0, \infty) \rightarrow \mathbf{R}$ is defined by $\varphi(q)=m c f(q)+m q^{a} g(q)+q^{a} h(q)$, and so

$$
\frac{\log \left(m \int_{1-\delta}^{1} q u^{q-1}(1-u)^{a} d u+h(q)\right)}{-\log q}=a-\frac{\log \varphi(q)}{\log q} .
$$

However, we clearly have $\left|q^{a} g(q)\right| \leq q^{a}(1-\delta)^{q} \rightarrow 0$ as $q \rightarrow \infty$ and $\left|q^{a} h(q)\right| \leq$ $q^{a}(1-\delta)^{q} \rightarrow 0$ as $q \rightarrow \infty$, and so $\varphi(q)=m c f(q)+m q^{a} g(q)+q^{a} h(q) \rightarrow m c$ as $q \rightarrow \infty$. The desired result follows from this and (3.3).

\section{Proof of Theorem 2.2}

The purpose of this section is to prove Theorem 2.2. We first introduce the following notation that will be used in this and the following sections. Namely, if $C$ is a subset of $\mathbf{R}^{m}$ and $r>0$, then we let $N_{r}(C)$ denote the largest number of pairwise disjoint balls of radii equal to $r$ and with centres in $C$, i.e.

$$
\begin{aligned}
N_{r}(C)=\sup \{\operatorname{card} I \mid & \left(B\left(x_{i}, r\right)\right)_{i \in I} \text { is a family of balls with: } \\
& \text { (1) } x_{i} \in C \text { for all } i ; \\
& \text { (2) } \left.B\left(x_{i}, r\right) \cap B\left(x_{j} r\right)=\varnothing \text { for all } i \neq j\right\} .
\end{aligned}
$$

It is well-known that lower and upper box dimension of $C$ are are given by

and

$$
\underline{\operatorname{dim}}_{\mathrm{B}} C=\liminf _{r \searrow 0} \frac{\log N_{r}(C)}{-\log r}
$$

$$
\overline{\operatorname{dim}}_{\mathrm{B}} C=\limsup _{r \searrow 0} \frac{\log N_{r}(C)}{-\log r} .
$$


respectively.

We now turn towards the proof of Theorem 2.2. We begin with the following small lemma.

Lemma 4.1. Let $\mu, \nu \in \mathcal{P}_{\text {co }}\left(\mathbf{R}^{d}\right)$. For each integer $n$ and $\varepsilon>0$, write

$$
\begin{aligned}
\Delta_{n, \varepsilon}=\left\{(x, y) \in \mathbf{R}^{d} \times \mathbf{R}^{d} \mid \overline{\operatorname{dim}}_{\mathrm{loc}}(\mu ; x)+\varepsilon \geq \frac{\log \mu(B(x, r))}{\log r} \text { for } 0<r<\frac{1}{n}\right. \\
\text { and } \left.\overline{\operatorname{dim}}_{\mathrm{loc}}(\nu ; y)+\varepsilon \geq \frac{\log \nu(B(y, r))}{\log r} \text { for } 0<r<\frac{1}{n}\right\} .
\end{aligned}
$$

Let $C$ be a Borel subset of $\operatorname{supp} \mu \times \operatorname{supp} \nu$. Then

$$
-\underline{\operatorname{dim}}_{\mathrm{B}}\left(\Delta_{n, \varepsilon} \cap C\right)+\sup _{(x, y) \in C}\left(\overline{\operatorname{dim}}_{\mathrm{loc}}(\mu ; x)+\overline{\operatorname{dim}}_{\mathrm{loc}}(\nu ; y)\right)+2 \varepsilon \geq 0
$$

for all positive integers $n$ and all $\varepsilon>0$.

Proof. For brevity write

$$
D=\sup _{(x, y) \in C}\left(\overline{\operatorname{dim}}_{\text {loc }}(\mu ; x)+\overline{\operatorname{dim}}_{\text {loc }}(\nu ; y)\right) .
$$

Fix a positive integer $n$ and $\varepsilon>0$. For each $0<r<\frac{1}{n}$, we can choose a subset $J_{r}$ of $\Delta_{n, \varepsilon} \cap C$ with

$$
\operatorname{card} J_{r}=N_{r}\left(\Delta_{n, \varepsilon} \cap C\right)
$$

and

$$
\text { the family }(B((x, y), r))_{(x, y) \in J_{r}} \text { consists of pairwise disjoint sets. }
$$

We now prove the following two claims.

Claim 1. For $0<r<\frac{1}{n}$, we have $N_{r}\left(\Delta_{n, \varepsilon} \cap C\right) \leq 2^{-\left(\frac{D}{2}+\varepsilon\right)} r^{-(D+2 \varepsilon)} \sum_{(x, y) \in J_{r}}(\mu \times$ $\nu) B((x, y), r)$. that

Proof of Claim 1. Noticing that $B\left(x, \frac{1}{\sqrt{2}} r\right) \times B\left(y, \frac{1}{\sqrt{2}} r\right) \subseteq B((x, y), r)$, we conclude

$$
\begin{aligned}
\sum_{(x, y) \in J_{r}}(\mu \times \nu) B((x, y), r) & \geq \sum_{(x, y) \in J_{r}}(\mu \times \nu)\left(B\left(x, \frac{1}{\sqrt{2}} r\right) \times B\left(y, \frac{1}{\sqrt{2}} r\right)\right) \\
& =\sum_{(x, y) \in J_{r}} \mu\left(B\left(x, \frac{1}{\sqrt{2}} r\right)\right) \nu\left(B\left(y, \frac{1}{\sqrt{2}} r\right)\right) .
\end{aligned}
$$

Next, we note that if $0<r<\frac{1}{n}$ and $(x, y) \in J_{r} \subseteq \Delta_{n, \varepsilon} \cap C \subseteq \Delta_{n, \varepsilon}$, then it follows from the definition of $\Delta_{n, \varepsilon}$ that $\mu\left(B\left(x, \frac{1}{\sqrt{2}} r\right)\right) \geq\left(\frac{1}{\sqrt{2}} r\right)^{\operatorname{dim}_{\mathrm{loc}}(\mu ; x)+\varepsilon}$ and $\nu\left(B\left(y, \frac{1}{\sqrt{2}} r\right)\right) \geq$ $\left(\frac{1}{\sqrt{2}} r\right)^{\overline{\operatorname{dim}}_{\mathrm{loc}}(\nu ; y)+\varepsilon}$. We deduce from this and (4.1) and (4.3) that

$$
\begin{aligned}
\sum_{(x, y) \in J_{r}}(\mu \times \nu) B((x, y), r) & \geq \sum_{(x, y) \in J_{r}}\left(\frac{1}{\sqrt{2}} r\right)^{\overline{\operatorname{dim}}_{\mathrm{loc}}(\mu ; x)+\varepsilon}\left(\frac{1}{\sqrt{2}} r\right)^{\overline{\operatorname{dim}}_{\mathrm{loc}}(\nu ; y)+\varepsilon} \\
& =\sum_{(x, y) \in J_{r}}\left(\frac{1}{\sqrt{2}} r\right)^{\overline{\operatorname{dim}}_{\mathrm{loc}}(\mu ; x)+\overline{\operatorname{dim}}_{\mathrm{loc}}(\nu ; y)+2 \varepsilon} \geq \sum_{(x, y) \in J_{r}}\left(\frac{1}{\sqrt{2}} r\right)^{D+2 \varepsilon} \\
& \geq 2^{-\left(\frac{D}{2}+\varepsilon\right)} r^{D+2 \varepsilon} \operatorname{card} J_{r} \geq 2^{-\left(\frac{D}{2}+\varepsilon\right)} r^{D+2 \varepsilon} N_{r}\left(\Delta_{n, \varepsilon} \cap C\right) .
\end{aligned}
$$

This completes the proof of Claim 1. 
Claim 2. For $0<r<\frac{1}{n}$, we have $\sum_{(x, y) \in J_{r}}(\mu \times \nu) B((x, y), r) \leq 1$.

Proof of Claim 2. Indeed, since the family $(B((x, y), r))_{(x, y) \in J_{r}}$ consists of pairwise disjoint sets (by (4.2)), we immediately conclude that $\sum_{(x, y) \in J_{r}}(\mu \times \nu) B((x, y), r)$ $=(\mu \times \nu)\left(\bigcup_{(x, y) \in J_{r}} B((x, y), r)\right) \leq 1$. This completes the proof of Claim 2.

By combining Claim 1 and Claim 2, we see that for $0<r<\frac{1}{n}$, we have

$$
N_{r}\left(\Delta_{n, \varepsilon} \cap C\right) \leq 2^{\frac{D}{2}+\varepsilon} r^{-(D+2 \varepsilon)} \sum_{(x, y) \in J_{r}}(\mu \times \nu) B((x, y), r) \leq 2^{\frac{D}{2}+\varepsilon} r^{-(D+2 \varepsilon)} .
$$

This inequality clearly implies that

$$
\underline{\operatorname{dim}}_{\mathrm{B}}\left(\Delta_{n, \varepsilon} \cap C\right) \leq \overline{\operatorname{dim}}_{\mathrm{B}}\left(\Delta_{n, \varepsilon} \cap C\right)=\limsup _{r \searrow 0} \frac{\log N_{r}\left(\Delta_{n, \varepsilon} \cap C\right)}{-\log r} \leq D+2 \varepsilon .
$$

We can now prove Theorem 2.2. The proof of Theorem 2.2 is divided into two parts. First we prove Theorem 2.2.(1) and then we prove Theorem 2.2.(2).

Proof of Theorem 2.2.(1). For the benefit of the reader we start by recalling the statement of Theorem 2.2.(1). Let $\mu, \nu \in \mathcal{P}_{\text {co }}\left(\mathbf{R}^{d}\right)$ and assume that

$$
d(\mu, \nu)=\sup _{\substack{x \in \operatorname{supp} \mu \\ y \in \operatorname{supp} \nu}}|x-y|=1 .
$$

Write $E=E(\mu, \nu)$. Then

$$
\limsup _{q \rightarrow \infty} \frac{M_{q}(\mu, \nu)}{-\log q} \leq-\operatorname{dim}_{\mathrm{H}} E+\sup _{(x, y) \in E}\left(\overline{\operatorname{dim}}_{\mathrm{loc}}(\mu ; x)+\overline{\operatorname{dim}}_{\mathrm{loc}}(\nu ; y)\right) .
$$

We will now prove (4.5). For a positive integer $n$ and $\varepsilon>0$, we let $\Delta_{n, \varepsilon}$ be defined as in Lemma 4.1, i.e.

$$
\begin{aligned}
\Delta_{n, \varepsilon}=\left\{(x, y) \in \mathbf{R}^{d} \times \mathbf{R}^{d} \mid \overline{\operatorname{dim}}_{\operatorname{loc}}(\mu ; x)+\varepsilon \geq \frac{\log \mu(B(x, r))}{\log r} \text { for } 0<r<\frac{1}{n}\right. \\
\left.\quad \text { and } \overline{\operatorname{dim}}_{\operatorname{loc}}(\nu ; y)+\varepsilon \geq \frac{\log \nu(B(y, r))}{\log r} \text { for } 0<r<\frac{1}{n}\right\} .
\end{aligned}
$$

For each positive integer $n$ and $\varepsilon>0$, we can find a positive number $r_{n, \varepsilon}$ such that if $0<r<r_{n, \varepsilon}$, then $\frac{\log N_{r}\left(\Delta_{n, \varepsilon} \cap E\right)}{-\log r} \geq \underline{\operatorname{dim}}_{\mathrm{B}}\left(\Delta_{n, \varepsilon} \cap E\right)-\varepsilon$, and so

$$
N_{r}\left(\Delta_{n, \varepsilon} \cap E\right) \geq r^{-\underline{\operatorname{dim}}_{\mathrm{B}}\left(\Delta_{n, \varepsilon} \cap E\right)+\varepsilon}
$$

for $0<r<r_{n, \varepsilon}$. Now, put $\delta_{n, \varepsilon}=\min \left(r_{n, \varepsilon}, \frac{1}{n}\right)$. Also, for each $u \in(0,1)$, we can choose a subset $I_{u, n, \varepsilon}$ of $\Delta_{n, \varepsilon} \cap E$ with

$$
\operatorname{card} I_{u, n, \varepsilon}=N_{\frac{1-u}{2}}\left(\Delta_{n, \varepsilon} \cap E\right)
$$

and

(4.8) the family $\left(B\left((x, y), \frac{1-u}{2}\right)\right)_{(x, y) \in I_{u, n, \varepsilon}}$ consists of pairwise disjoint sets.

It now follows from Lemma 3.2 that the is a function $h_{n, \varepsilon}:(0, \infty) \rightarrow \mathbf{R}$ such that

$$
M_{q}(\mu, \nu)=I_{n, \varepsilon}(q)+h_{n, \varepsilon}(q)
$$

where

$$
I_{n, \varepsilon}(q)=\int_{1-\delta_{n, \varepsilon}}^{1} q u^{q-1}(\mu \times \nu)\left\{(x, y) \in \mathbf{R}^{d} \times \mathbf{R}^{d}|| x-y \mid \geq u\right\} d u
$$


and $\left|h_{n, \varepsilon}(q)\right| \leq\left(1-\delta_{n, \varepsilon}\right)^{q}$ for all $q>0$. We will now estimate $I_{n, \varepsilon}(q)$. This is done in Claim 1 below. For brevity, we write

$$
\begin{aligned}
D & =\sup _{(x, y) \in E}\left(\overline{\operatorname{dim}}_{\text {loc }}(\mu ; x)+\overline{\operatorname{dim}}_{\text {loc }}(\nu ; y)\right), \\
s_{n, \varepsilon} & =\underline{\operatorname{dim}}_{\mathrm{B}}\left(\Delta_{n, \varepsilon} \cap E\right), \quad a_{n, \varepsilon}=-s_{n, \varepsilon}+D+3 \varepsilon,
\end{aligned}
$$

in Claim 1 and below.

Claim 1. We have $I_{n, \varepsilon}(q) \geq m \int_{1-\delta_{n, \varepsilon}}^{1} q u^{q-1}(1-u)^{a_{n, \varepsilon}} d u$ where $m=2^{-\varepsilon}(2 \sqrt{2})^{-(D+2 \varepsilon)}$.

Proof of Claim 1. We first note that

$$
\bigcup_{(x, y) \in I_{u, n, \varepsilon}}\left(B\left(x, \frac{1-u}{2}\right) \times B\left(y, \frac{1-u}{2}\right)\right) \subseteq\left\{(v, w) \in \mathbf{R}^{d} \times \mathbf{R}^{d}|| v-w \mid \geq u\right\} ;
$$

indeed, if $(x, y) \in I_{u, n, \varepsilon} \subseteq \Delta_{n, \varepsilon} \cap E \subseteq E$ and $(v, w) \in B\left(x, \frac{1-u}{2}\right) \times B\left(y, \frac{1-u}{2}\right)$, then $1=d(\mu, \nu)=|x-y| \leq|x-v|+|v-w|+|w-y|=\frac{1-u}{2}+|v-w|+\frac{1-u}{2}=|v-w|+1-u$, whence $|v-w| \geq u$. This proves (4.10). It follows from (4.10) that

$$
\begin{aligned}
I_{n, \varepsilon}(q) & =\int_{1-\delta_{n, \varepsilon}}^{1} q u^{q-1}(\mu \times \nu)\left\{(v, w) \in \mathbf{R}^{d} \times \mathbf{R}^{d}|| v-w \mid \geq u\right\} d u \\
& \geq \int_{1-\delta_{n, \varepsilon}}^{1} q u^{q-1}(\mu \times \nu)\left(\bigcup_{(x, y) \in I_{u, n, \varepsilon}}\left(B\left(x, \frac{1-u}{2}\right) \times B\left(y, \frac{1-u}{2}\right)\right)\right) d u .
\end{aligned}
$$

Noticing that $B\left((x, y), \frac{1-u}{2}\right) \subseteq B\left(x, \frac{1-u}{2}\right) \times B\left(y, \frac{1-u}{2}\right)$ for all $(x, y) \in \mathbf{R}^{d} \times \mathbf{R}^{d}$ and all $u \in(0,1)$, we conclude from (4.11) that

$$
I_{n, \varepsilon}(q) \geq \int_{1-\delta_{n, \varepsilon}}^{1} q u^{q-1}(\mu \times \nu)\left(\bigcup_{(x, y) \in I_{u, n, \varepsilon}} B\left((x, y), \frac{1-u}{2}\right)\right) d u .
$$

Next, since it follows from (4.8) that the family $\left(B\left((x, y), \frac{1-u}{2}\right)\right)_{(x, y) \in I_{u, n, \varepsilon}}$ consists of pairwise disjoint sets, we conclude that

$$
(\mu \times \nu)\left(\bigcup_{(x, y) \in I_{u, n, \varepsilon}} B\left((x, y), \frac{1-u}{2}\right)\right)=\sum_{(x, y) \in I_{u, n, \varepsilon}}(\mu \times \nu) B\left((x, y), \frac{1-u}{2}\right),
$$

and we therefore deduce from (4.12) that

$$
I_{n, \varepsilon}(q) \geq \int_{1-\delta_{n, \varepsilon}}^{1} q u^{q-1} \sum_{(x, y) \in I_{u, n, \varepsilon}}(\mu \times \nu) B\left((x, y), \frac{1-u}{2}\right) d u .
$$

We now notice that $B\left(x, \frac{1}{\sqrt{2}} \frac{1-u}{2}\right) \times B\left(y, \frac{1}{\sqrt{2}} \frac{1-u}{2}\right) \subseteq B\left((x, y), \frac{1-u}{2}\right)$ for all $(x, y) \in$ $\mathbf{R}^{d} \times \mathbf{R}^{d}$ and all $u \in(0,1)$. This and (4.13) imply that

$$
\begin{aligned}
I_{n, \varepsilon}(q) & \geq \int_{1-\delta_{n, \varepsilon}}^{1} q u^{q-1} \sum_{(x, y) \in I_{u, n, \varepsilon}}(\mu \times \nu)\left(B\left(x, \frac{1}{\sqrt{2}} \frac{1-u}{2}\right) \times B\left(y, \frac{1}{\sqrt{2}} \frac{1-u}{2}\right)\right) d u \\
& =\int_{1-\delta_{n, \varepsilon}}^{1} q u^{q-1} \sum_{(x, y) \in I_{u, n, \varepsilon}} \mu\left(B\left(x, \frac{1}{\sqrt{2}} \frac{1-u}{2}\right)\right) \nu\left(B\left(y, \frac{1}{\sqrt{2}} \frac{1-u}{2}\right)\right) d u .
\end{aligned}
$$


We now observe that if $(x, y) \in I_{u, n, \varepsilon}$ and $u \in\left(1-\delta_{n, \varepsilon}, 1\right)$, then $(x, y) \in I_{u, n, \varepsilon} \subseteq$ $\Delta_{n, \varepsilon} \cap E \subseteq \Delta_{n, \varepsilon}$ and $\frac{1}{\sqrt{2}} \frac{1-u}{2} \leq 1-u \leq \delta_{n, \varepsilon} \leq \frac{1}{n}$, whence

$$
\begin{aligned}
& \mu\left(B\left(x, \frac{1}{\sqrt{2}} \frac{1-u}{2}\right)\right) \geq\left(\frac{1}{\sqrt{2}} \frac{1-u}{2}\right) \overline{\operatorname{dim}}_{\mathrm{loc}}(\mu ; x)+\varepsilon, \\
& \nu\left(B\left(x, \frac{1}{\sqrt{2}} \frac{1-u}{2}\right)\right) \geq\left(\frac{1}{\sqrt{2}} \frac{1-u}{2}\right) \overline{\operatorname{dim}}_{\mathrm{loc}}(\nu ; y)+\varepsilon .
\end{aligned}
$$

We deduce from this and (4.7) and (4.14) that

$$
\begin{aligned}
I_{n, \varepsilon}(q) & \geq \int_{1-\delta_{n, \varepsilon}}^{1} q u^{q-1} \sum_{(x, y) \in I_{u, n, \varepsilon}} \mu\left(B\left(x, \frac{1}{\sqrt{2}} \frac{1-u}{2}\right)\right) \nu\left(B\left(y, \frac{1}{\sqrt{2}} \frac{1-u}{2}\right)\right) d u \\
& \geq \int_{1-\delta_{n, \varepsilon}}^{1} q u^{q-1} \sum_{(x, y) \in I_{u, n, \varepsilon}}\left(\frac{1}{\sqrt{2}} \frac{1-u}{2}\right)^{\overline{\operatorname{dim}}_{\mathrm{loc}}(\mu ; x)+\varepsilon}\left(\frac{1}{\sqrt{2}} \frac{1-u}{2}\right)^{\overline{\operatorname{dim}}_{\mathrm{loc}}(\nu ; y)+\varepsilon} d u \\
& =\int_{1-\delta_{n, \varepsilon}}^{1} q u^{q-1} \sum_{(x, y) \in I_{u, n, \varepsilon}}\left(\frac{1}{\sqrt{2}} \frac{1-u}{2}\right)^{\overline{\operatorname{dim}}_{\mathrm{loc}}(\mu ; x)+\overline{\operatorname{dim}}_{\mathrm{loc}}(\nu ; y)+2 \varepsilon} d u \\
& \geq \int_{1-\delta_{n, \varepsilon}}^{1} q u^{q-1} \sum_{(x, y) \in I_{u, n, \varepsilon}}\left(\frac{1}{\sqrt{2}} \frac{1-u}{2}\right)^{D+2 \varepsilon} d u \\
& =\int_{1-\delta_{n, \varepsilon}}^{1} q u^{q-1}\left(\frac{1}{\sqrt{2}} \frac{1-u}{2}\right)^{D+2 \varepsilon} \operatorname{card} I_{u, n, \varepsilon} d u \\
& =\int_{1-\delta_{n, \varepsilon}}^{1} q u^{q-1}\left(\frac{1}{\sqrt{2}} \frac{1-u}{2}\right)^{D+2 \varepsilon} N_{\frac{1-u}{2}}\left(\Delta_{n, \varepsilon} \cap E\right) d u .
\end{aligned}
$$

Finally, we observe that if $u \in\left(1-\delta_{n, \varepsilon}, 1\right)$, then $1-u \leq \delta_{n, \varepsilon} \leq r_{n, \varepsilon}$, and (4.6) therefore shows that

$$
N_{\frac{1-u}{2}}\left(\Delta_{n, \varepsilon} \cap E\right) \geq\left(\frac{1-u}{2}\right)^{-\underline{\operatorname{dim}}_{\mathrm{B}}\left(\Delta_{n, \varepsilon} \cap E\right)+\varepsilon} \geq 2^{-\varepsilon}(1-u)^{-\underline{\operatorname{dim}}_{\mathrm{B}}\left(\Delta_{n, \varepsilon} \cap E\right)+\varepsilon} .
$$

This and (4.15) imply that

$$
\begin{aligned}
I_{n, \varepsilon}(q) & \geq \int_{1-\delta_{n, \varepsilon}}^{1} q u^{q-1}\left(\frac{1}{\sqrt{2}} \frac{1-u}{2}\right)^{D+2 \varepsilon} 2^{-\varepsilon}(1-u)^{-\underline{\operatorname{dim}}_{\mathrm{B}}\left(\Delta_{n, \varepsilon} \cap E\right)+\varepsilon} d u \\
& =m \int_{1-\delta_{n, \varepsilon}}^{1} q u^{q-1}(1-u)^{a_{n, \varepsilon}} d u .
\end{aligned}
$$

This completes the proof of Claim 1.

Combining Claim 1 and (4.9) yields

$$
M_{q}(\mu, \nu)=I_{n, \varepsilon}(q)+h_{n, \varepsilon}(q) \geq m \int_{1-\delta_{n, \varepsilon}}^{1} q u^{q-1}(1-u)^{a_{n, \varepsilon}} d u+h_{n, \varepsilon}(q),
$$

for all positive integers $n$ and all $\varepsilon>0$, where $\left|h_{n, \varepsilon}(q)\right| \leq\left(1-\delta_{n, \varepsilon}\right)^{q}$ for all $q>0$, and $a_{n, \varepsilon}=-s_{n, \varepsilon}+D+3 \varepsilon=-\underline{\operatorname{dim}}_{\mathrm{B}}\left(\Delta_{n, \varepsilon} \cap E\right)+D+3 \varepsilon \geq \varepsilon>0$ since $-\underline{\operatorname{dim}}_{\mathrm{B}}\left(\Delta_{n, \varepsilon} \cap E\right)+$ $D+2 \varepsilon \geq 0$ by Lemma 4.1. Since $\left|h_{n, \varepsilon}(q)\right| \leq\left(1-\delta_{n, \varepsilon}\right)^{q}$ for all $q>0$, and $a_{n, \varepsilon}>0$, it follows from Lemma 3.4 and (4.16) that

$$
\begin{aligned}
\limsup _{q \rightarrow \infty} \frac{\log M_{q}(\mu, \nu)}{-\log q} & \leq \lim _{q \rightarrow \infty} \frac{\log \left(m \int_{1-\delta_{n, \varepsilon}}^{1} q u^{q-1}(1-u)^{a_{n, \varepsilon}} d u+h_{n, \varepsilon}(q)\right)}{-\log q} \\
& =a_{n, \varepsilon}=-\underline{\operatorname{dim}}_{\mathrm{B}}\left(\Delta_{n, \varepsilon} \cap E\right)+D+3 \varepsilon
\end{aligned}
$$


for all positive integers $n$ and all $\varepsilon>0$. Using the fact that $\operatorname{dim}_{\mathrm{H}}\left(\Delta_{n, \varepsilon} \cap E\right) \leq$ $\underline{\operatorname{dim}}_{\mathrm{B}}\left(\Delta_{n, \varepsilon} \cap E\right)$, we therefore conclude that

$$
\limsup _{q \rightarrow \infty} \frac{\log M_{q}(\mu, \nu)}{-\log q} \leq-\underline{\operatorname{dim}}_{\mathrm{H}}\left(\Delta_{n, \varepsilon} \cap E\right)+D+3 \varepsilon
$$

for all positive integers $n$ and all $\varepsilon>0$.

Since (4.17) holds for all $n$, we now deduce that

$$
\limsup _{q \rightarrow \infty} \frac{\log M_{q}(\mu, \nu)}{-\log q} \leq-\sup _{n} \operatorname{dim}_{\mathrm{H}}\left(\Delta_{n, \varepsilon} \cap E\right)+D+3 \varepsilon .
$$

However, it is clear that $\bigcup_{n} \Delta_{n, \varepsilon}=\mathbf{R}^{d} \times \mathbf{R}^{d}$, whence $\sup _{n} \operatorname{dim}_{\mathrm{H}}\left(\Delta_{n, \varepsilon} \cap E\right)=$ $\operatorname{dim}_{\mathrm{H}}\left(\bigcup_{n}\left(\Delta_{n, \varepsilon} \cap E\right)\right)=\operatorname{dim}_{\mathrm{H}} E$, and (4.18) therefore implies that

$$
\limsup _{q \rightarrow \infty} \frac{\log M_{q}(\mu, \nu)}{-\log q} \leq-\operatorname{dim}_{\mathrm{H}} E+D+3 \varepsilon .
$$

Finally, letting $\varepsilon \searrow 0$ gives the desired result.

Proof of Theorem 2.2.(2). For the benefit of the reader we start by recalling the statement of Theorem 2.2.(2). Let $\mu, \nu \in \mathcal{P}_{\text {co }}\left(\mathbf{R}^{d}\right)$ and assume that

$$
d(\mu, \nu)=\sup _{\substack{x \in \operatorname{supp} \mu \\ y \in \operatorname{supp} \nu}}|x-y|=1 .
$$

Write $E=E(\mu, \nu)$. Then

$$
\limsup _{q \rightarrow \infty} \frac{M_{q}(\mu, \nu)}{-\log q} \leq \inf _{(x, y) \in E}\left(\overline{\operatorname{dim}}_{\mathrm{loc}}(\mu ; x)+\overline{\operatorname{dim}}_{\mathrm{loc}}(\nu ; y)\right) .
$$

We will now prove (4.20). Fix $\varepsilon>0$ and fix $(x, y) \in E$. We can find $\delta_{\varepsilon}>0$ such that if $0<r<\delta_{\varepsilon}$, then

$$
\overline{\operatorname{dim}}_{\mathrm{loc}}(\mu ; x)+\varepsilon \geq \frac{\log \mu B(x, r)}{\log r}, \quad \overline{\operatorname{dim}}_{\mathrm{loc}}(\nu ; y)+\varepsilon \geq \frac{\log \nu B(y, r)}{\log r} .
$$

It now follows from Lemma 3.2 that the is a function $h_{\varepsilon}:(0, \infty) \rightarrow \mathbf{R}$ such that

$$
M_{q}(\mu, \nu)=I_{\varepsilon}(q)+h_{\varepsilon}(q)
$$

where

$$
I_{\varepsilon}(q)=\int_{1-\delta_{\varepsilon}}^{1} q u^{q-1}(\mu \times \nu)\left\{(v, w) \in \mathbf{R}^{d} \times \mathbf{R}^{d}|| v-w \mid \geq u\right\} d u
$$

and $\left|h_{\varepsilon}(q)\right| \leq(1-\delta)^{q}$ for all $q>0$. We will now estimate $I_{\varepsilon}(q)$. This is done in Claim 1 below. For brevity, we write

$$
D=\overline{\operatorname{dim}}_{\mathrm{loc}}(\mu ; x)+\overline{\operatorname{dim}}_{\mathrm{loc}}(\nu ; y), \quad a_{\varepsilon}=D+3 \varepsilon,
$$

in Claim 1 and below.

Claim 1. We have $I_{\varepsilon}(q) \geq m \int_{1-\delta_{\varepsilon}}^{1} q u^{q-1}(1-u)^{a_{\varepsilon}} d u$ where $m=2^{-a_{\varepsilon}}$.

Proof of Claim 1. We first note that

$$
B\left(x, \frac{1-u}{2}\right) \times B\left(y, \frac{1-u}{2}\right) \subseteq\left\{(v, w) \in \mathbf{R}^{d} \times \mathbf{R}^{d}|| v-w \mid \geq u\right\} ;
$$

indeed, since $(x, y) \in E$, we deduce that $|x-y|=d(\mu, \nu)=1$, and we therefore conclude that if $(v, w) \in B\left(x, \frac{1-u}{2}\right) \times B\left(y, \frac{1-u}{2}\right)$, then $1=|x-y| \leq|x-v|+|v-w|+$ 
$|w-y|=\frac{1-u}{2}+|v-w|+\frac{1-u}{2}=|v-w|+1-u$, whence $|v-w| \geq u$. This proves (4.23). It follows from (4.23) that

$$
\begin{aligned}
I_{\varepsilon}(q) & =\int_{1-\delta_{\varepsilon}}^{1} q u^{q-1}(\mu \times \nu)\left\{(v, w) \in \mathbf{R}^{d} \times \mathbf{R}^{d}|| v-w \mid \geq u\right\} d u \\
& \geq \int_{1-\delta_{\varepsilon}}^{1} q u^{q-1}(\mu \times \nu)\left(B\left(x, \frac{1-u}{2}\right) \times B\left(y, \frac{1-u}{2}\right)\right) d u \\
& =\int_{1-\delta_{\varepsilon}}^{1} q u^{q-1} \mu\left(B\left(x, \frac{1-u}{2}\right)\right) \nu\left(B\left(y, \frac{1-u}{2}\right)\right) d u .
\end{aligned}
$$

We now observe that if $u \in\left(1-\delta_{\varepsilon}, 1\right)$, then $\frac{1-u}{2} \leq 1-u \leq \delta_{\varepsilon}$, whence (using

$$
\mu\left(B\left(x, \frac{1-u}{2}\right)\right) \geq\left(\frac{1-u}{2}\right)^{\overline{\operatorname{dim}}_{\mathrm{loc}}(\mu ; x)+\varepsilon}, \quad \nu\left(B\left(x, \frac{1-u}{2}\right)\right) \geq\left(\frac{1-u}{2}\right)^{\overline{\operatorname{dim}}_{\mathrm{loc}}(\nu ; y)+\varepsilon} .
$$

We deduce from this and (4.24) that

$$
I_{\varepsilon}(q) \geq \int_{1-\delta_{\varepsilon}}^{1} q u^{q-1}\left(\frac{1-u}{2}\right)^{\overline{\operatorname{dim}}_{\mathrm{loc}}(\mu ; x)+\varepsilon}\left(\frac{1-u}{2}\right)^{\overline{\operatorname{dim}}_{\mathrm{loc}}(\nu ; y)+\varepsilon} d u=m \int_{1-\delta_{\varepsilon}}^{1} q u^{q-1}(1-u)^{a_{\varepsilon}} d u .
$$

This completes the proof of Claim 1.

Combining Claim 1 and (4.22) yields

$$
M_{q}(\mu, \nu)=I_{\varepsilon}(q)+h_{\varepsilon}(q) \geq m \int_{1-\delta_{\varepsilon}}^{1} q u^{q-1}(1-u)^{a_{\varepsilon}} d u+h_{\varepsilon}(q),
$$

where $\left|h_{\varepsilon}(q)\right| \leq\left(1-\delta_{\varepsilon}\right)^{q}$ for all $q>0$, and $a_{\varepsilon}=D+2 \varepsilon \geq 2 \varepsilon>0$ since clearly $D \geq 0$. Since $\left|h_{\varepsilon}(q)\right| \leq\left(1-\delta_{\varepsilon}\right)^{q}$ for all $q>0$, and $a_{\varepsilon}>0$, it follows from Lemma 3.4 and (4.22) that

$$
\begin{aligned}
\limsup _{q \rightarrow \infty} \frac{\log M_{q}(\mu, \nu)}{-\log q} & \leq \lim _{q \rightarrow \infty} \frac{\log \left(m \int_{1-\delta_{\varepsilon}}^{1} q u^{q-1}(1-u)^{a_{\varepsilon}} d u+h_{\varepsilon}(q)\right)}{-\log q} \\
& =a_{\varepsilon}=\overline{\operatorname{dim}}_{\mathrm{loc}}(\mu ; x)+\overline{\operatorname{dim}}_{\mathrm{loc}}(\nu ; y)+2 \varepsilon .
\end{aligned}
$$

Finally, taking infimum over all $(x, y) \in E$ and letting $\varepsilon \searrow 0$ gives the desired result.

\section{Proof of Theorem 2.4}

The purpose of this section is to prove Theorem 2.4. We start by recalling the following well-known covering lemma.

Lemma 5.1. Let $A$ be a subset of $\mathbf{R}^{m}$ and let $r>0$. Then there is a finite or countable subset $I$ of $A$ such that the family $(B(x, r))_{x \in I}$ of balls with centres in $I$ and radii equal to $r$ satisfying the following two conditions:

(1) If $x, y \in I$ and $x \neq y$, then $B(x, r) \cap B(y, r)=\varnothing$.

(2) We have $\bigcup_{x \in A} B(x, r) \subseteq \bigcup_{x \in I} B(x, 5 r)$.

Proof. The result is proven in [Ma, Theorem 2.1].

We can now prove Theorem 2.4. 
Proof of Theorem 2.4. For the benefit of the reader we start by recalling the statement of Theorem 2.4. Let $\mu, \nu \in \mathcal{P}_{\text {co }}\left(\mathbf{R}^{d}\right)$ and assume that

$$
d(\mu, \nu)=\sup _{\substack{x \in \operatorname{supp} \mu \\ y \in \operatorname{supp} \nu}}|x-y|=1 \text {. }
$$

Write $E=E(\mu, \nu)$. Assume that $\mu$ and $\nu$ satisfy the extremal covering condition, i.e. there a positive constant $k_{0}>0$ and a number $0<r_{0}<1$ such that $E_{r} \subseteq B\left(E_{0}, k_{0} r\right)$ for all $r$ with $0<r<r_{0}$ where $E_{r}$ is defined in (2.4). Then

$$
\liminf _{q \rightarrow \infty} \frac{\log M_{q}(\mu, \nu)}{-\log q} \geq-\overline{\operatorname{dim}}_{\mathrm{B}} E+\liminf _{r \searrow 0} \inf _{(x, y) \in E} \frac{\log (\mu \times \nu) B((x, y), r)}{\log r} .
$$

We will now prove (5.1). For brevity write

$$
D=\liminf _{r \searrow 0} \inf _{(x, y) \in E} \frac{\log (\mu \times \nu) B((x, y), r)}{\log r}, \quad s=\overline{\operatorname{dim}}_{\mathrm{B}} E .
$$

We must now prove that $\liminf _{q \rightarrow \infty} \frac{\log M_{q}(\mu, \nu)}{-\log q} \geq-s+D$. This inequality is clearly satisfied if $-s+D \leq 0$, and we will therefore assume that $-s+D>0$.

Fix $\varepsilon$ with $0<\varepsilon<\frac{1}{2}(-s+D)$. Recall, the definition of the box dimensions in Section 4. In particular, recall that if $C$ is a subset of $\mathbf{R}^{m}$ and $r>0$, then we let $N_{r}(C)$ denotes the largest number of pairwise disjoint balls of radii equal to $r$ and with centres in $C$. It follows from the definitions of the upper box dimension and the number $D$ that we can find $\delta_{\varepsilon}$ with $0<\delta_{\varepsilon}<r_{0}$ such that $\frac{\log N_{r}(E)}{-\log r} \leq \overline{\operatorname{dim}}_{\mathrm{B}} E+\varepsilon$ for all $r \leq \delta_{\varepsilon}$ and $\inf _{(x, y) \in E} \frac{\log (\mu \times \nu) B((x, y), r)}{\log r} \geq D-\varepsilon$ for all $r \leq 5 k_{0} \delta_{\varepsilon}$. In particular, this implies that

$$
N_{r}(E) \leq r^{-\overline{\operatorname{dim}}_{\mathrm{B}} E-\varepsilon}
$$

for all $r \leq \delta_{\varepsilon}$, and

$$
(\mu \times \nu) B((x, y), r) \leq r^{D-\varepsilon}
$$

for all $(x, y) \in E$ and all $r \leq 5 k_{0} \delta_{\varepsilon}$.

Next, we conclude from Lemma 3.2 that the is a function $h_{\varepsilon}:(0, \infty) \rightarrow \mathbf{R}$ such that

$$
M_{q}(\mu, \nu)=I_{\varepsilon}(q)+h_{\varepsilon}(q)
$$

where

$$
I_{\varepsilon}(q)=\int_{1-\delta_{\varepsilon}}^{1} q u^{q-1}(\mu \times \nu)\left\{(x, y) \in \mathbf{R}^{d} \times \mathbf{R}^{d}|| x-y \mid \geq u\right\} d u
$$

and $\left|h_{\varepsilon}(q)\right| \leq\left(1-\delta_{\varepsilon}\right)^{q}$ for all $q>0$. We will now estimate $I_{\varepsilon}(q)$. This is done in Claim 1 below. For brevity we write

$$
a_{\varepsilon}=-s+D-2 \varepsilon
$$

in Claim 1 below.

Claim 1. We have $I_{\varepsilon}(q) \leq m \int_{1-\delta_{\varepsilon}}^{1} q u^{q-1}(1-u)^{a_{\varepsilon}} d u$ where $m=\left(5 k_{0}\right)^{D-\varepsilon} k_{0}^{-s-\varepsilon}$.

Proof of Claim 1. We first note that if $u \in\left(1-\delta_{\varepsilon}, 1\right)$, then $1-u<\delta_{\varepsilon}<r_{0}$, whence $E_{1-u} \subseteq B\left(E_{0}, k_{0}(1-u)\right)=B\left(E, k_{0}(1-u)\right.$ ) (because $E_{0}=E$ ), and so

$$
E_{1-u} \subseteq B\left(E, k_{0}(1-u)\right)=\bigcup_{(x, y) \in E} B\left((x, y), k_{0}(1-u)\right) .
$$


Applying Lemma 5.1 to the family $\left\{B\left((x, y), k_{0}(1-u)\right) \mid(x, y) \in E\right\}$ (i.e. the family of balls with centres in $E$ and radii equal to $k(1-u)$ ), we conclude that there is a finite or countable subset $I_{u}$ of $E$ such that the family $\left\{B\left((x, y), k_{0}(1-u)\right)\right.$ $\left.(x, y) \in I_{u}\right\}$ of balls with centres in $I_{u}$ and radii equal to $k_{0}(1-u)$ satisfies the following two conditions:

(1) If $\left(x_{1}, y_{1}\right),\left(x_{2}, y_{2}\right) \in I_{u}$ and $\left(x_{1}, y_{1}\right) \neq\left(x_{2}, y_{2}\right)$, then $B\left(\left(x_{1}, y_{1}\right), k_{0}(1-u)\right) \cap$ $B\left(\left(x_{2}, y_{2}\right), k_{0}(1-u)\right)=\varnothing$

(2) We have $\bigcup_{(x, y) \in E} B\left((x, y), k_{0}(1-u)\right) \subseteq \bigcup_{(x, y) \in I_{u}} B\left((x, y), 5 k_{0}(1-u)\right)$.

Combining (5.5) and Condition (2), we now deduce that

$$
\begin{aligned}
I_{\varepsilon}(q) & =\int_{1-\delta_{\varepsilon}}^{1} q u^{q-1}(\mu \times \nu)\left\{(x, y) \in \mathbf{R}^{d} \times \mathbf{R}^{d}|| x-y \mid \geq u\right\} d u \\
& =\int_{1-\delta_{\varepsilon}}^{1} q u^{q-1}(\mu \times \nu)\left(E_{1-u}\right) d u \\
& \leq \int_{1-\delta_{\varepsilon}}^{1} q u^{q-1}(\mu \times \nu)\left(\bigcup_{(x, y) \in E} B\left((x, y), k_{0}(1-u)\right)\right) d u \\
& \leq \int_{1-\delta_{\varepsilon}}^{1} q u^{q-1}(\mu \times \nu)\left(\bigcup_{(x, y) \in I_{u}} B\left((x, y), 5 k_{0}(1-u)\right)\right) d u \\
& \leq \int_{1-\delta_{\varepsilon}}^{1} q u^{q-1} \sum_{(x, y) \in I_{u}}(\mu \times \nu)\left(B\left((x, y), 5 k_{0}(1-u)\right)\right) d u .
\end{aligned}
$$

However, if $u \in\left(1-\delta_{\varepsilon}, 1\right)$ and $(x, y) \in I_{u}$, then $(x, y) \in E$ and $5 k_{0}(1-u) \leq 5 k_{0} \delta_{\varepsilon}$, and it therefore follows from $(5.3)$ that $(\mu \times \nu)\left(B\left((x, y), 5 k_{0}(1-u)\right)\right) \leq\left(5 k_{0}(1-u)\right)^{D-\varepsilon}$. This and (5.6) imply that

$$
\begin{aligned}
I_{\varepsilon}(q) & \leq \int_{1-\delta_{\varepsilon}}^{1} q u^{q-1} \sum_{(x, y) \in I_{u}}\left(5 k_{0}(1-u)\right)^{D-\varepsilon} d u \\
& =\left(5 k_{0}\right)^{D-\varepsilon} \int_{1-\delta_{\varepsilon}}^{1} q u^{q-1}(1-u)^{D-\varepsilon} \operatorname{card} I_{u} d u .
\end{aligned}
$$

Next, we observe that it follows from Condition (1) that the family $\left(B\left((x, y), k_{0}(1-\right.\right.$ $u))_{(x, y) \in I_{u}}$ consists of pairwise disjoint balls with centres in $I_{u} \subseteq E$ and radii equal to $k_{0}(1-u)$. This clearly implies that

$$
\operatorname{card} I_{u} \leq N_{k_{0}(1-u)}(E) .
$$

Combining (5.7) and (5.8) now gives

$$
I_{\varepsilon}(q) \leq\left(5 k_{0}\right)^{D-\varepsilon} \int_{1-\delta_{\varepsilon}}^{1} q u^{q-1}(1-u)^{D-\varepsilon} N_{k_{0}(1-u)}(E) d u .
$$

We also notice that if $u \in\left(1-\delta_{\varepsilon}, 1\right)$, then $k(1-u) \leq k_{0} \delta_{\varepsilon}$, and (5.2) therefore implies that

$$
N_{k_{0}(1-u)}(E) \leq\left(k_{0}(1-u)\right)^{-\overline{\operatorname{dim}}_{\mathrm{B}} E-\varepsilon}
$$


We deduce from (5.9) and (5.10) that

$$
\begin{aligned}
I_{\varepsilon}(q) & \leq\left(5 k_{0}\right)^{D-\varepsilon} \int_{1-\delta_{\varepsilon}}^{1} q u^{q-1}(1-u)^{D-\varepsilon}\left(k_{0}(1-u)\right)^{-\overline{\operatorname{dim}}_{\mathrm{B}} E-\varepsilon} d u \\
& =m \int_{1-\delta_{\varepsilon}}^{1} q u^{q-1}(1-u)^{a_{\varepsilon}} d u .
\end{aligned}
$$

This completes the proof of Claim 1.

Combining Claim 1 and (5.4) yields

$$
M_{q}(\mu, \nu)=I_{\varepsilon}(q)+h_{\varepsilon}(q) \leq m \int_{1-\delta_{\varepsilon}}^{1} q u^{q-1}(1-u)^{a_{\varepsilon}} d u+h_{\varepsilon}(q)
$$

where $\left|h_{\varepsilon}(q)\right| \leq\left(1-\delta_{\varepsilon}\right)^{q}$ for all $q>0$, and $a_{\varepsilon}=-s+D-2 \varepsilon>0$ (because $\left.0<\varepsilon<\frac{1}{2}(-s+D)\right)$. Since $\left|h_{\varepsilon}(q)\right| \leq\left(1-\delta_{\varepsilon}\right)^{q}$ for all $q>0$, and $a_{\varepsilon}>0$, it follows from Lemma 3.4 and (5.11) that

$$
\begin{aligned}
\liminf _{q \rightarrow \infty} \frac{\log M_{q}(\mu, \nu)}{-\log q} & \geq \lim _{q \rightarrow \infty} \frac{\log \left(m \int_{1-\delta_{\varepsilon}}^{1} q u^{q-1}(1-u)^{a_{\varepsilon}} d u+h(q)\right)}{-\log q} \\
& =a_{\varepsilon}=-\overline{\operatorname{dim}}_{\mathrm{B}} E+D-2 \varepsilon .
\end{aligned}
$$

Finally, letting $\varepsilon \searrow 0$ gives the desired result.

Acknowledgements. The author thanks an anonymous referee for having read the manuscript carefully and providing many useful comments.

\section{References}

[CaMa] Cawley, R., and R. D. Mauldin: Multifractal decomposition of Moran fractals. Adv. Math. 92, 1992, 196-236.

[Fa1] FALCONER, K. J.: Fractal geometry - Mathematical foundations and applications. - John Wiley, Chichester, 1990

[Fa2] Falconer, K. J.: Techniques in fractal geometry. - Wiley, 1997.

[HaJeKaPrSh] Halsey, T. C., M. H. Jensen, L. P. Kadanoff, I. Procaccia, and B. J. SHRAIMAN: Fractal measures and their singularities: The characterization of strange sets. - Phys. Rev. A 33, 1986, 1141-1151

[HeTh] Hengartner, W., and R. Theodorescu: Concentration functions. - Probab. Math. Statist. 20, Academic Press, New York-London, 1973.

[Ma] Mattila, P.: Geometry of sets and measures in Euclidean spaces. Fractals and rectifiability - Cambridge Stud. Adv. Math. 44, Cambridge Univ. Press, Cambridge, 1995.

[Ol] Olver, F. W. J.: Asymptotics and special functions - Computer Science and Applied Mathematics, Academic Press, New York-London, 1974.

$[\mathrm{Pe}] \quad$ Pesin, Y.: Dimension theory in dynamical systems. Contemporary Views and Applications. - The Univ. of Chicago Press, 1997.

[Ré1] RÉNYi, A.: Some fundamental questions of information theory. - Magyar Tud. Akad. Mat. Fiz. Oszt. Közl 10, 1960, 251-282

[Ré2] RÉnyi, A.: On measures of entropy and information. - In: Proceedings 4th Berkeley Symposium on Mathematical Statistics and Probability 1960, Univ. of California Press, Berkeley, 1961, 547-561. 